\title{
A Survey of Network Lifetime Maximization Techniques in Wireless Sensor Networks
}

\author{
Halil Yetgin, Kent Tsz Kan Cheung, Mohammed El-Hajjar, Senior member, IEEE and Lajos Hanzo, Fellow, IEEE
}

\begin{abstract}
Emerging technologies, such as the Internet of things, smart applications, smart grids and machine-to-machine networks stimulate the deployment of autonomous, selfconfiguring, large-scale wireless sensor networks (WSNs). Efficient energy utilization is crucially important in order to maintain a fully operational network for the longest period of time possible. Therefore, network lifetime (NL) maximization techniques have attracted a lot of research attention owing to their importance in terms of extending the flawless operation of battery-constrained WSNs. In this paper, we review the recent developments in WSNs, including their applications, design constraints and lifetime estimation models. Commencing with the portrayal of rich variety definitions of NL design objective used for WSNs, the family of NL maximization techniques is introduced and some design guidelines with examples are provided to show the potential improvements of the different design criteria.
\end{abstract}

\section{NOMENCLATURE}

$\begin{array}{ll}\text { AWGN } & \text { Additive White Gaussian Noise } \\ \text { BER } & \text { Bit Error Rate } \\ \text { CFs } & \text { Constraint Functions } \\ \text { CSI } & \text { Channel State Information } \\ \text { DN } & \text { Destination Node } \\ \text { ED } & \text { Energy Dissipation } \\ \text { EH } & \text { Energy Harvesting } \\ \text { IoT } & \text { Internet of Things } \\ \text { MAC } & \text { Medium Access Control } \\ \text { NL } & \text { Network Lifetime } \\ \text { OFs } & \text { Objectives Functions } \\ \text { QoS } & \text { Quality of Service } \\ \text { REI } & \text { Residual Energy Information } \\ \text { RL } & \text { Route Lifetime } \\ \text { SINR } & \text { Signal-to-Interference-Plus-Noise Ratio } \\ \text { SN } & \text { Source Node } \\ \text { SNR } & \text { Signal-to-Noise Ratio } \\ \text { TDMA } & \text { Time-Division Multiple Access } \\ \text { WSNs } & \text { Wireless Sensor Networks }\end{array}$

\section{INTRODUCTION}

A wireless sensor network (WSN) is constituted by spatially distributed autonomous devices communicating wirelessly,

This research has been funded by the Republic of Turkey Ministry of National Education. H. Yetgin is with Bitlis Eren university, Merkez/Bitlis, Turkey (e-mail: hyetgin@beu.edu.tr), K. T. K. Cheung, M. El-Hajjar, and L. Hanzo are with the School of Electronics and Computer Science, University of Southampton, Southampton, SO17 1BJ, UK (e-mail: $\{$ meh,lh\}@ecs.soton.ac.uk).

L. Hanzo would also like to gratefully acknowledge the financial support of the European Research Council's Senior Research Fellow Grant Beam-Me-Up and of the Royal Society's Wolfson Award.

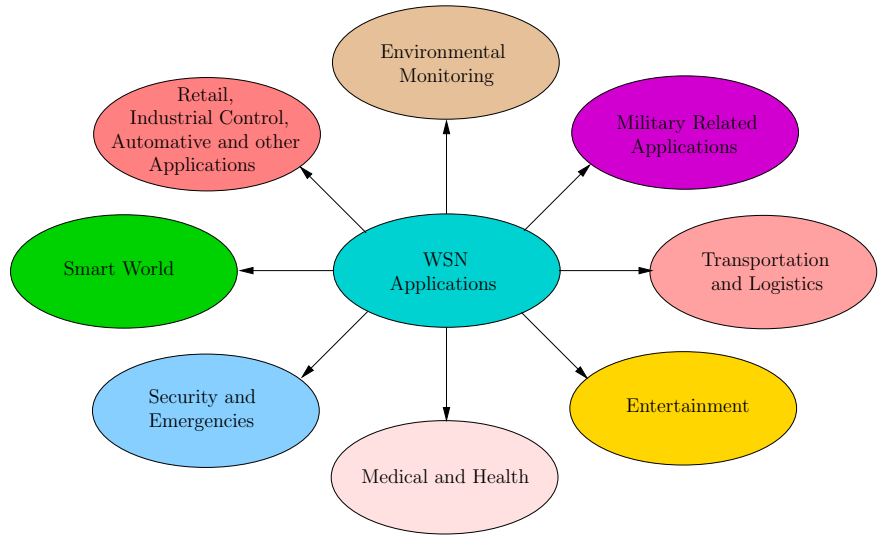

Fig. 1. The taxonomy of the WSN applications.

gathering information and detecting certain events of significance in the physical and environmental conditions. Each of these devices is capable of concurrently sensing, processing and communicating. Having these capabilities on a sensor device offers a vast number of compelling applications [1]-[5], as illustrated in Fig. 1. For example, one of the oldest application areas of WSNs is found in environmental monitoring, ranging from the tracking herds of animals to the monitoring hard-to-reach areas. Military battlefields also constitute a potential application of WSNs, especially in inaccessible or hostile territory, where WSNs may be indispensable for the detection of snipers, intruders and for tracking their activity. Additionally, the deployment of WSNs can be very useful for improving logistics, where tackling the challenges in managing goods that are being transported can preserve their quality by monitoring the temperature of containers, just to mention a few.

As another example, WSNs can be used for improving the gaming experience by enhancing the interactions between the physical world and virtual world using wearable and implantable camera sensors. Medical and health applications form another important set of WSN applications enabling carers to monitor the conditions of patients either in hospital or in elder people's home. Radiation level control, explosive gas level and leakage detection, as well as restricted area control also form part of the potential security and emergency applications.

In the Internet of things (IoT) era, there is a plethora of applications using WSNs [6], in smart cities and other smart environments, in remote metering and smart water provision, in efficient agriculture as well as smart farming and so on [7]. Many other sophisticated WSN applications have been proposed in the literature for improving the quality of human life, 
such as supply-chain control for retail purposes, remote control of home appliances, industrial factory automation, automotive, rail-, and air-traffic control as well as disaster control. The contributions of this paper are summarized as follows.

1) We provide a compact classification of smart WSN applications, considering the recent advances.

2) A comprehensive list of the design constraints of WSNs is provided.

3) A broad overview of network lifetime (NL) definitions is offered.

4) We provide a critical appraisal of the state-of-the-art NL maximization techniques, including their objective functions (OFs), constraint functions (CFs), optimization tools and optimality.

5) After discussing all the relevant NL maximization contributions in the literature, we will provide generic design examples for maximizing the NL of WSNs.

The rest of this paper is organized as illustrated in Fig. 2. We provide a comprehensive list of definitions of the NL design objective in Section II. Then, the typical design constraints of WSNs are described in Section III, followed by the portrayal of NL maximization techniques in Section IV. Finally, in Section V we provide a summary section, including our conclusions and generic design criteria examples in the interest of maximizing the NL. We will close with some future research directions.

\section{Network Lifetime Design Objective}

A physically tangible definition of the NL design objective can be formulated as the total amount of time during which the network is capable of maintaining its full functionality and/or of achieving particular objectives during its operation, as exemplified in [31] ${ }^{1}$ and [32]. Moreover, the NL is a crucial metric in terms of providing the system designer with well-informed decisions for the sake of maintaining the desired network performance and the QoS in WSNs, where the sensor nodes usually rely on a limited battery capacity, unless they have direct mains supply. Moreover, in realistic applications, replenishing the battery energy of the sensors or replacing the sensors is usually impractical. Therefore, the NL is constrained by the battery of the individual sensors in the WSN considered [1], [2]. However, the definition of NL may vary depending on the specific application, on the objective function and on the network topology considered. Specifically, the authors of [15], [16] and [14] defined the expiration of the $\mathrm{NL}$ as the time instant at which a certain number of nodes in the network depleted their batteries. As a further example, the NL was defined in [33] as the lifetime of the specific sensor node associated with the highest energy consumption rate, whereas the authors of [10], [8] and [12] considered the lifetime of the network to be expired at the particular instant, when the first node's battery was depleted. The NL in [8] was also defined as the instant, when the first data collection failure

\footnotetext{
${ }^{1}$ This paper is a more recent contribution on NL definitions than [31] from 2009. However, [31] is mostly focused on the definitions of NL, including their own parameterized NL definition. In this paper, we have provided a comprehensive survey of NL maximization techniques, which has not been disseminated in the literature.
}

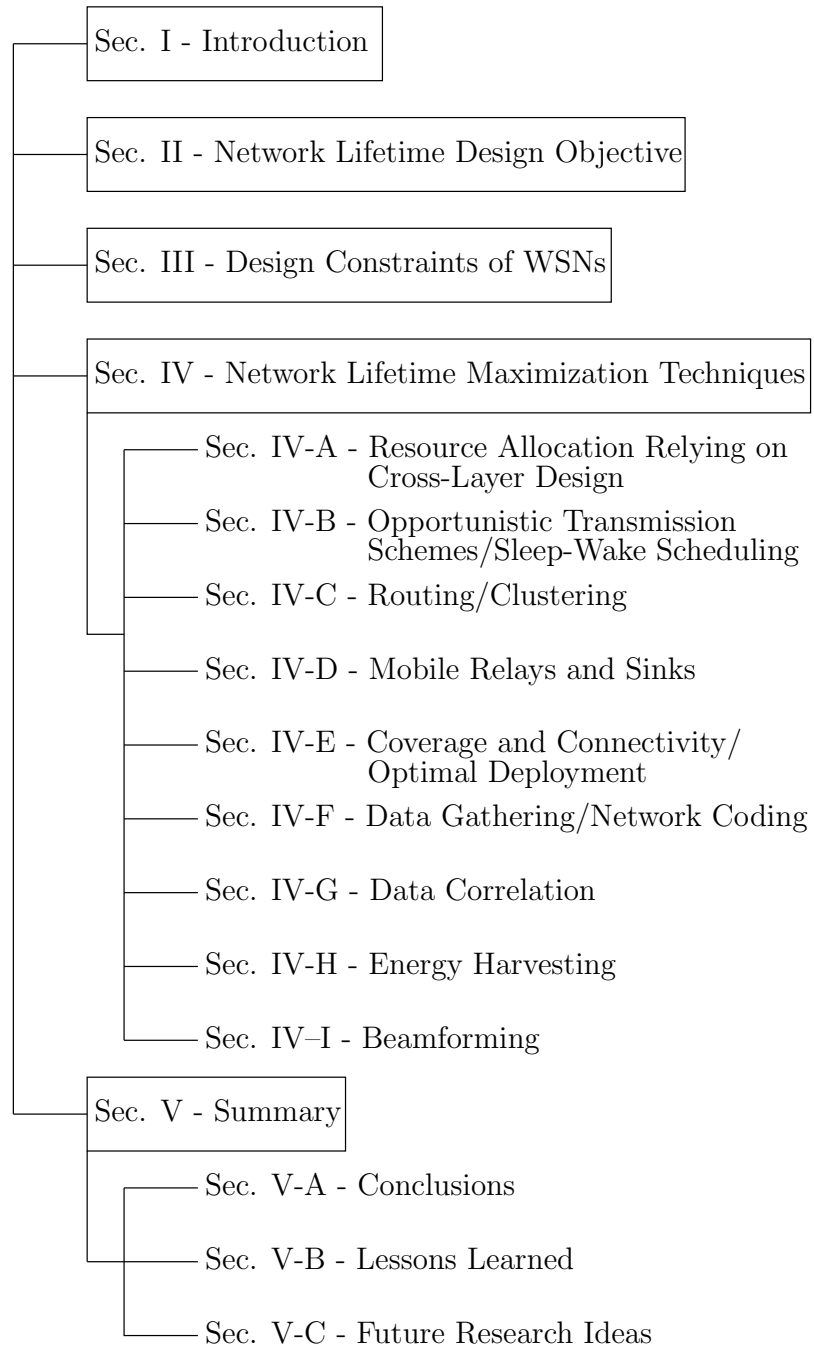

Fig. 2. The structure overview of this paper.

occurred. Note that there are various alternative NL definitions, which were discussed in [3], [8], [10], [13], [31], [34]-[36]. In Table I, we provide a comprehensive list of the NL definitions considered in the literature. We classify the NL definitions into four categories, namely the family of node-lifetime based NL, coverage and connectivity based NL, transmission based NL and parameterized NL definitions. More explicitly, nodelifetime based NL definitions are dependent on the longevity of the sensors, while the coverage and connectivity based NL definitions are based on providing coverage for a specific target area and/or on guaranteeing a particular quality of the connectivity. The family of transmission based NL definitions relies on the delivery of information, for example on data collection failure, on event detection ratio, on sensory information estimation, on data reception failure at the sink, on the SNR as the QoS requirement and on a broad range of other transmission-characteristic based NL definitions. A NL definition may also be parameterized by the probability of node availability, by the quality of coverage and connectivity and so on. Finally, we categorise the different NL objectives as "NL-1, NL-2, NL-3,..., NL-14" in Table I, which are then used to specify the exact NL design objective used in the literature 
TABLE I

THE CLASSIFICATION OF DEFINITIONS OF THE NL DESIGN OBJECTIVE.

\begin{tabular}{|c|c|c|}
\hline NL Category & NL Order & Examples \\
\hline \multirow{4}{*}{ Node-lifetime } & NL-1 & $\begin{array}{l}\text { The earliest time instant at which any of the sensor nodes in the network fully depletes its } \\
\text { battery [8]-[13]. }\end{array}$ \\
\hline & NL-2 & $\begin{array}{l}\text { The time instant, beyond which only a certain fraction of operational nodes remains in the } \\
\text { network [14]-[16]. }\end{array}$ \\
\hline & NL-3 & The time, at which the first cluster head fully discharges its battery [17]. \\
\hline & NL-4 & The time, when all the sensor nodes in the network fully deplete their battery [18]. \\
\hline \multirow{2}{*}{$\begin{array}{l}\text { Coverage and } \\
\text { connectivity }\end{array}$} & NL-5 & $\begin{array}{l}\text { The time duration, for which the target area is covered by at least } k \text { nodes, which was } \\
\text { termed as the } k \text {-coverage in [19]. }\end{array}$ \\
\hline & NL-6 & $\begin{array}{l}\text { The time, until a specific target area [20]-[22] or the entire area [23], [24] is not covered } \\
\text { by at least a single sensor node. }\end{array}$ \\
\hline \multirow[t]{2}{*}{ based NL } & NL-7 & $\begin{array}{l}\text { The total amount of time, beyond which either the coverage or the packet delivery ratio } \\
\text { falls below a certain threshold [25]. }\end{array}$ \\
\hline & NL-8 & The time duration up to the moment, when the coverage is lost [26]. \\
\hline \multirow{5}{*}{$\begin{array}{l}\text { Transmission } \\
\text { based NL }\end{array}$} & NL-9 & The time, within which a certain amount of information has been transmitted [27]. \\
\hline & NL-10 & $\begin{array}{l}\text { The time duration up to the moment, when the network becomes incapable of maintaining } \\
\text { a reasonable event detection ratio [18]. }\end{array}$ \\
\hline & NL-11 & $\begin{array}{l}\text { The number of sensory information estimation task cycles achieved before the network } \\
\text { becomes nonoperational [28]. }\end{array}$ \\
\hline & NL-12 & $\begin{array}{l}\text { The time instant, when the last report is delivered to the sink [8], [29]. More explicitly, the } \\
\text { time-span until the specific instant, when the first data collection failure occurred. }\end{array}$ \\
\hline & NL-13 & $\begin{array}{l}\text { The longest time period, over which the QoS constraint, such as the signal-to-noise } \\
\text { ratio (SNR) requirement is satisfied [30]. }\end{array}$ \\
\hline Parameterized & NL-14 & $\begin{array}{l}\text { In [31], a parameterized NL definition was stipulated, including the above-mentioned } \\
\text { common definitions, such as node availability, coverage, connectivity, data collection and } \\
\text { so on. }\end{array}$ \\
\hline NL & & $\begin{array}{l}\text { This NL definition can be used for most of the applications, since the required objective } \\
\text { can be incorporated into or discarded from the formulation of the NL definition. }\end{array}$ \\
\hline
\end{tabular}

surveyed in Tables III-IX.

\section{Design CONSTRAints OF WSNS}

The above-mentioned applications have been designed for accomplishing a specific objective or a desired task. Therefore, as illustrated in Fig. 3, there are several design constraints, denoted by "Const.-1, Const.-2, Const.-3,..., Const.6", which necessitate the careful consideration of the WSN deployment depending on the application requirements and on the objectives to be achieved [1]-[3], [5]. Observe in Fig. 3 that the particular choice of the communication medium affects the design of the communication protocols, because different radio spectral bands require different communication configurations, including the transmit power, the effective transmission distance, the presence or absence of line-of-sight propagation, the interference levels encountered, and so on. Similarly, once the carrier frequency has been determined, the related channel characteristics [37] play a significant role in predetermining the attainable performance of the application considered. Additionally, as illustrated in Fig. 3, the cost of each sensor device is also an important design factor in terms of determining the total cost of the WSN, since the application considered may require the scattering of thousands of sensor devices in a specific field, which also requires careful consideration of the network size and the topology in order to maximize the NL [38], [39]. Hence, indepth studies have been dedicated to minimizing the total cost of the WSN, while providing the maximum grade of connectivity and coverage quality in the interest of NL maximization [40], [41]. Fig. 3 captures the main design constraints of WSNs at a glance, demonstrating that the battery capacity, computing and storage capabilities constitute precious limited resources, which the design of WSNs hinges on. Fig. 3 suggests that the network topology [35], [42]-[44] is another crucial aspect influencing the design of WSNs, since it is often conceived for a particular application, which indeed affects all the salient 


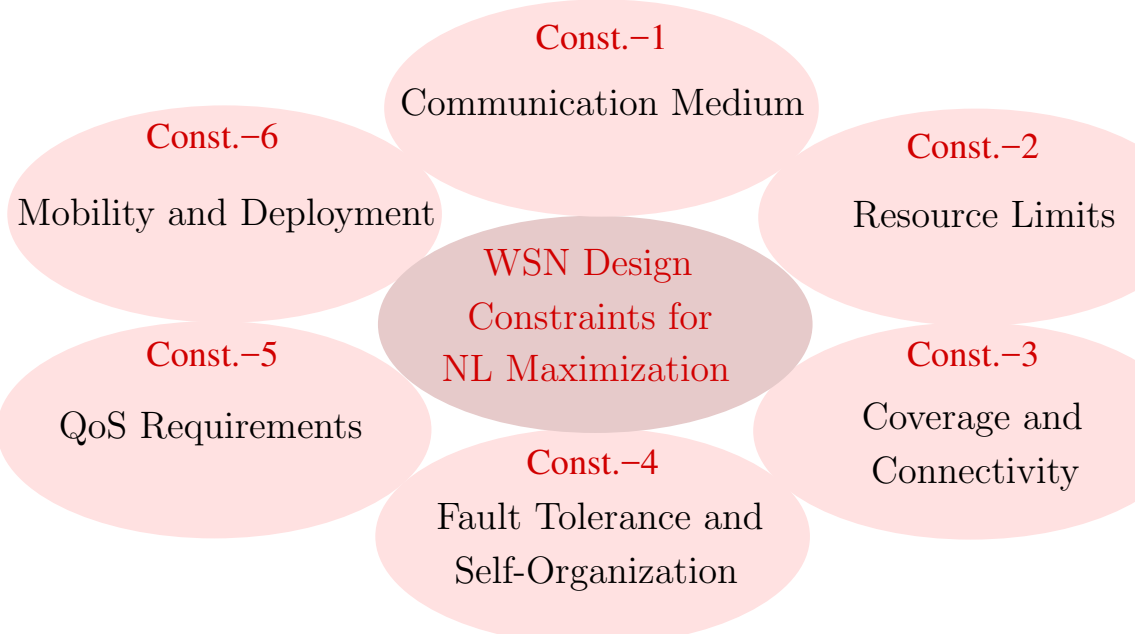

Fig. 3. The design constraints of WSNs for maximizing the NL.

network characteristics, such as the delay, the capacity, the routing complexity, the energy consumption and the NL, which are constrained by the network resources.

Additionally, it is crucial to maintain a high grade of connectivity and coverage quality, which is facilitated by the appropriate density of nodes. To elaborate a little further, an excessive node-density generates excessive traffic conveying correlated data, whilst an insufficient density degrades the coverage quality. Coverage quality has been extensively studied in the context of WSNs [26], [40], [41], which is crucial for the sake of maintaining seamless connectivity. Explicitly, the coverage quality and the grade of connectivity influences the choice of data gathering methods and routing algorithms designed for achieving the desired quality of service (QoS) requirements, as indicated in Fig. 3.

Moreover, the data gleaned from a particular sensor node may be corrupted by the hostile, error-prone wireless channel, hence it is important to verify and if necessary, to correct the information relayed to the sink node for increasing the attainable reliability [37], [45]-[48]. Additionally, in the event of node failure, the network still has to remain operational and robust [49], where maintaining fault tolerance is also of high significance. Specifically, in large-scale WSN deployments, self-organization and self-configuration assist the network in replacing the failing sensor nodes without perturbing the entire application [50], as illustrated in Fig. 3. It also has to be resilient against denial-of-service (DoS) attacks and must be resistant to eavesdropping [3], [5].

Additionally, application-specific QoS requirements, such as the latency, energy dissipation (ED), lifetime, bit error rate (BER), throughput, interference levels, time synchronization accuracy and data redundancy have to be taken into account during the deployment of the WSN in order to guarantee the seamless operation of the application considered [36], [51], as illustrated in Fig. 3. From a physical layer point of view, maximizing the throughput whilst concurrently reducing the BER may be feasible, but only at an increased implementational complexity and at a commensurately increased ED necessitated by sophisticated signal processing [52], as illustrated in Fig. 3. More explicitly, the attainable capacity of a wireless link strictly depends on the signal power, on the noise and on the interference levels at the receiver [9], [11]. Since the channel conditions are time-variant, maintaining the required BER can be a challenging task in a low-power WSN [36], as indicated in Fig. 3. Additionally, in densely populated WSNs, the data observed by the adjacent sensors may be correlated [53], which may result in an inefficient exploitation of the resources, especially when the NL maximization is a key objective to be considered.

The specific deployment strategies of WSNs [38], [39] substantially affect the characteristics of the network, such as the sensor node density, the specific sensor locations, the anticipated degree of network dynamics and the longevity of WSNs. Similarly, the grade and the nature of mobility also has a significant impact on the degree of network dynamics as well as on the NL, which affects the design of both the routing protocols and of the associated distributed algorithms [3], [5], [29], [54], [55]. Explicitly, the above-mentioned design factors crucially depend on the mobility characteristics, as presented in Fig. 3. Therefore, the design factors of self-configuration, self-organization, robustness, reliability and fault tolerance play a significant role in constructing an adaptive and scalable WSN, where the longevity of the network is a crucial objective to be accomplished [49].

The lifetime of a WSN represents the total amount of time, over which the network remains operational and hence supports the application considered [11], [36]. Therefore, observe in Fig. 3 that the network's lifetime is one of the most important design factors in WSNs, since all the abovementioned design constraints can only be met, if the network is operational. Explicitly, in this treatise we specifically focused our attention on the NL as our design objective, while the stateof-the-art in NL maximization techniques is also surveyed.

Finally, in the following sections we explicitly specify the design constraints of Fig. 3, denoted by "Const.-1, Const.2, Const.-3,..., Const.-6", that are used in the state-of-theart literature in the column of "Constraint Function (CF)" of Tables III-IX. Therefore, one can readily observe the NL design objective as well as the design constraints utilized in the state-of-the-art literature of Tables III-IX using Fig. 3 and 
Table I.

\section{Network Lifetime Maximization Techniques}

There are several NL maximization techniques in the literature, as classified in Fig. 5. Each of them may consider a different NL definition and a different objective function, where the NL definition may also vary depending on the application, on the particular objective and on the network topology considered. Observe in Fig. 5 that resource allocation, opportunistic transmission schemes, sleep-wake scheduling, routing, clustering, mobile relays and sinks, coverage and connectivity, optimal deployment, data gathering, network coding, data correlation, energy harvesting and beamforming are the most important techniques we highlight in this part of the paper. Therefore, we classify these techniques in Fig. 5, where the NL is maximized using a particular type of technique from the literature. Some papers are related to multiple NL maximization techniques. However, here we classify the papers according to their main context and focus.

The classification in Fig. 5 is created based on the most influential papers in the field of the WSNs that focus on the network lifetime maximization as their objective function. We have searched through the lavish WSN literature and cited the most influential papers in the field, which tend to be the most highly cited research papers. In Table II, we list the papers we used to define our classification together with the total number of citations of these papers and then we list the number of citations for the most and least cited papers. The least cited papers tend to be the newest papers published during the last year. As mentioned before, these papers are selected as the most influential papers while focusing our attention on the papers, where the objective was to maximize the NL. This is how the NL maximization techniques illustrated in Fig. 5 were selected.

In the following, we will discuss each NL maximization technique in detail based on Fig. 5.

\section{A. Resource Allocation Relying on Cross-Layer Design}

Resource allocation is one of the most important and perhaps the most frequently investigated NL maximization techniques in the literature [9], [11], [37], [44], [47], [128]. Resource allocation operations typically rely on the crosslayer optimization of various cross-layer design constraints, including the transmission reliability, routing, power control, scheduling, optimal node-deployment, throughput maximization, estimation quality and rate adaptation, which indeed form part of the design constraints in WSNs, as presented in Fig. 3. Hence, resource allocation may be combined with various NL maximization techniques, since all NL maximization techniques rely on some resource allocation algorithm. For example, Hoesel et al. [56] proposed a cross-layer approach for jointly optimizing the MAC and routing layer in order to maximize the NL, where the MAC layer sets the sensors to either their active- or inactive-mode and the routing layer aims for finding energy-efficient routes in the face of a dynamic topology. In [47], Kwon et al. investigated the NL maximization problem of WSNs, which jointly considers the physical layer, the MAC layer and the routing layer in conjunction with the end-to-end transmission success probability constraint. The authors of [47] demonstrated that the joint optimization of power control, retransmission control and routing optimization is capable of significantly improving the NL compared to suboptimal algorithms. Another resource allocation approach was proposed for NL maximization by Xu et al. [37], examining the conflicting design objectives, including the transmit rate, delivery reliability and NL using an optimization framework imposing time-varying channel capacity, reliability and energy constraints and demonstrated that the selection of the suitable weights for each of these objectives is crucial for the sake of meeting the desired application performance.

Additionally, the authors of [9] considered the joint optimal design of the transmit rate, power and link scheduling for the sake of NL maximization in an interferencelimited WSN communicating over an additive white Gaussian noise (AWGN) channel and demonstrated the benefit of multihop routing, traffic-load balancing, interference management and spatial reuse in extending the NL. Similarly, in [58] the cross-layer operation of the link layer, MAC layer and routing was invoked for maximizing the NL considering the transmitter's circuit ED in a WSN communicating over an AWGN channel. Another cross-layer optimization technique was employed in [57] for illustrating the trade-off between NL maximization and application performance. As a further advance, the authors of [59] investigated the trade-off between the energy consumption and application-layer performance exploiting the interplay between network lifetime maximization and rate allocation problems with the aid of cross-layer operation in WSNs. Additionally, Wang et al. [44], [128] advocated a cross-layer approach in order to minimize the ED and to maximize the NL of a WSN composed of multiple sources and a single sink, where power allocation, link scheduling and routing problems were jointly optimized. A similar study was performed in [60], formulating the network lifetime maximization problem as a joint power, rate and scheduling problem subjected to rate distortion constraints, capacity constraints of the links, energy constraint of the sensor batteries and delay constraint of the encoded data arriving at the sink node.

In [11], we formulated the NL maximization problem as a convex optimization problem in Equations (1)-(7) encompassing the routing, scheduling, as well as the transmission rate and power allocation operations for transmission over an AWGN channel, where $z$ is the reciprocal of the NL. The links that are active in time slot $n$ are denoted by the set $\mathcal{L}_{n}$, while $\mathbf{s}=\left[s_{1}, 0, \cdots, 0,-s_{1}\right]^{T}$ is the source rate vector. The first and last elements of the source vector are nonzero, and the remaining elements are set to zero, because the first node is the source node ( $\mathrm{SN}$ ) and the last node is the destination node (DN), while the other nodes act as relay nodes. The variables of the optimization problem are $z$, $Q_{l, n}$ and $r_{l, n}$ for $l \in \mathcal{L}_{n}, n=1, \cdots, N$. The vector of the rate variables associated with time slot $n$ is given by $\mathbf{r}_{n}=$ $\left[r_{l_{1,2}, n}, r_{l_{2,3}, n}, \cdots, r_{l_{V-1, V}, n}\right]^{T}$. Furthermore, we denote the 


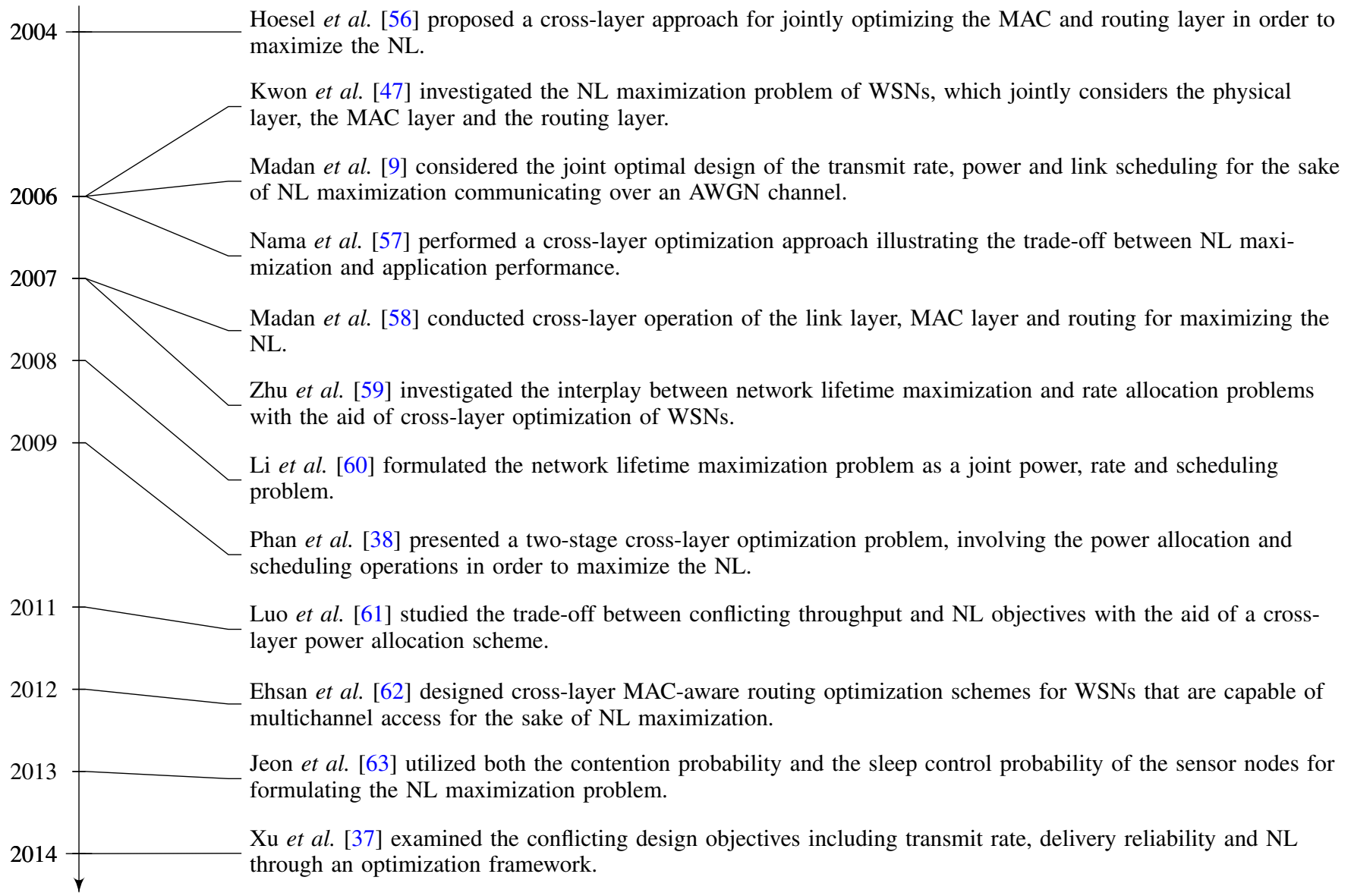

Fig. 4. Timeline of resource allocation techniques that maximize the lifetime of WSNs.

power amplifier's efficiency as $(1-\alpha)$ [135]. Equation (2) guarantees the flow-conservation, which physically implies the delivery of information generated at the SN to the DN. The specific resources, such as the transmit rate is constrained by the power in Eq. (3). The energy-conservation constraint is given by Eq. (4), which allows each node to dissipate at most the initial amount of battery energy. Equation (5) sets the constraint on the transmission power of a given node. Exploiting (1)-(7), we derived the closed-form equations of the instantaneous transmission rate and the power variables in (8)-(9) with the aid of the Karush-Kuhn-Tucker optimality conditions of [136] and Lagrangian constrained optimization. We obtained optimal solutions for the transmit rate and for the power variables using Gauss-Seidel and gradient ascent algorithms. For more details, the motivated readers are referred to [11].

Another cross-layer approach conceived for maximizing the $\mathrm{NL}$ was proposed in [62], where MAC-aware routing optimization schemes were designed for WSNs that are capable of multichannel access. A different approach to NL maximization was introduced in [63], where both the contention probability and the sleep control probability of the sensor nodes was utilized for formulating the NL maximization problem, while maintaining both the throughput and the signal to interference plus noise ratio (SINR) requirements.
An optimal control approach was invoked for maximizing the NL with the aid of a carefully selected routing probability [12], where all the sensors were configured to deplete their energy exactly at the same time. Additionally, Phan et al. [38] presented a two-stage cross-layer optimization problem, where the first stage involves maximizing the number of sensor nodes deployed for the existing WSN and the second stage includes the power allocation and scheduling operations in order to maximize the NL. A similar cross-layer design approach was proposed in [64] by adopting the constraints of the joint routing and MAC layers in order to maximize the NL. The authors of [61] studied the trade-off between conflicting throughput and NL objectives with the aid of a cross-layer power allocation scheme and demonstrated that an optimal choice of transmit power is essential in the interest of achieving a high throughput and a high NL. The authors of [65] investigated the impact of the transmit rate on the NL for both single-hop and multi-hop transmission scenarios by exploiting the interplay between the estimation accuracy of the channel as well as the data transmitted and energyefficiency. This is mainly because increasing the transmit rate degrades the NL, but on the other hand improves the estimation quality of the data transmitted. In this section, we outlined the major contributions in the context of resource allocation, as illustrated in Fig. 4. The OFs, the constraints, 


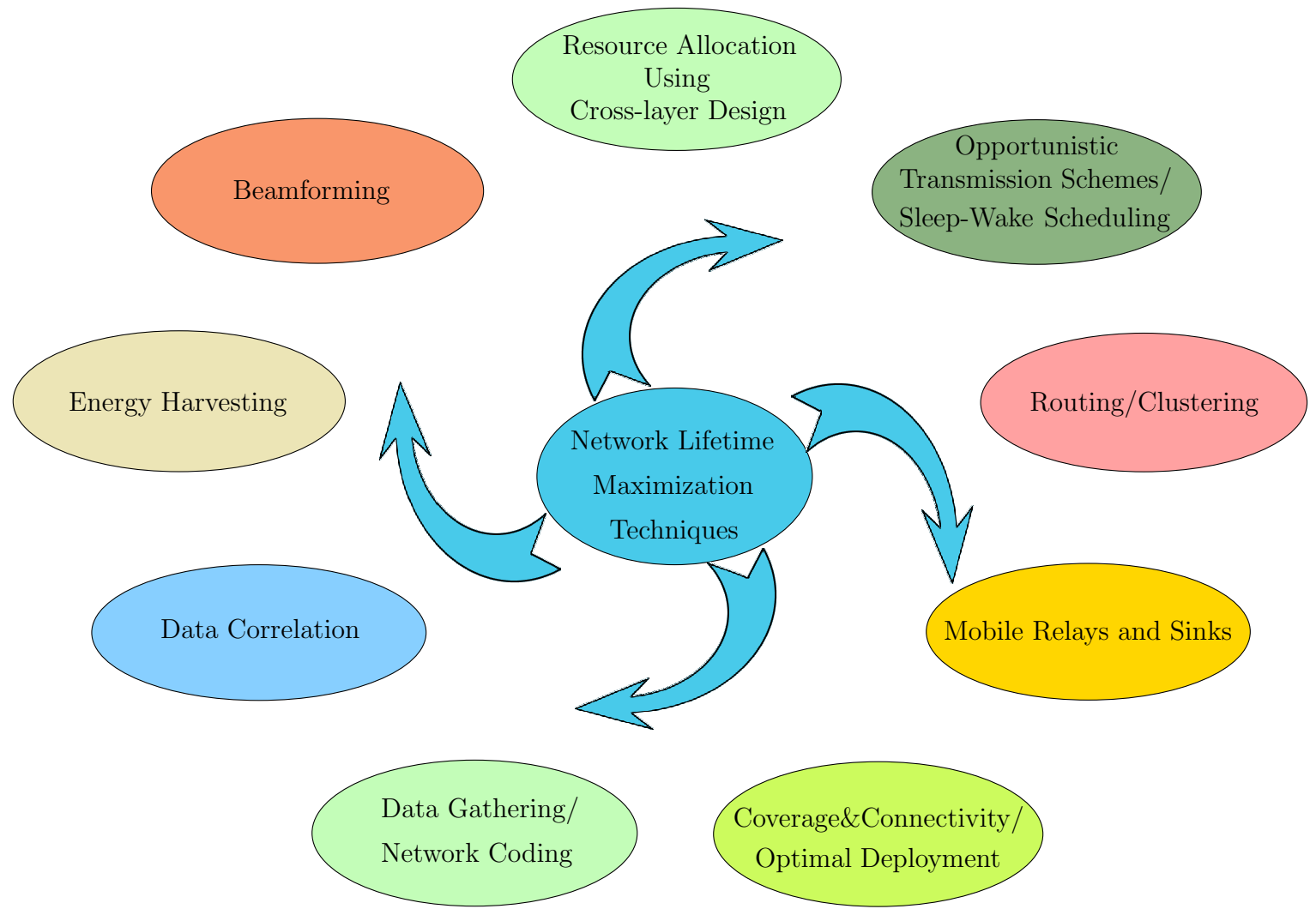

Fig. 5. The classification of the NL maximization techniques.

TABLE II

REFERENCES SELECTION CRITERIA IN THE CONTEXT OF NETWORK LIFETIME MAXIMISATION OF WSNS.

\begin{tabular}{|c|c|c|c|c|}
\hline Classification & Papers & $\begin{array}{l}\text { Total number of } \\
\text { citations }\end{array}$ & $\begin{array}{l}\text { Number of cita- } \\
\text { tions for the most } \\
\text { cited paper }\end{array}$ & $\begin{array}{l}\text { Number of cita- } \\
\text { tions for the least } \\
\text { cited paper }\end{array}$ \\
\hline $\begin{array}{l}\text { Resource allocation using } \\
\text { cross-layer design }\end{array}$ & 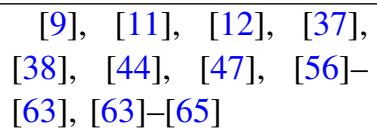 & 1250 & 401 & 12 \\
\hline $\begin{array}{l}\text { Opportunistic } \\
\text { transmission } \\
\text { schemes/Sleep-wake } \\
\text { scheduling }\end{array}$ & {$[8],[56],[63],[66]-[76]$} & 1609 & 433 & 14 \\
\hline Routing/Clustering & $\begin{array}{l}{[12],[28],[43],[62],} \\
{[71],[72],[77]-[86]}\end{array}$ & 5530 & 2027 & 16 \\
\hline Mobile relays and sinks & $\begin{array}{l}{[29],[54],[55],[87]-} \\
{[92]}\end{array}$ & 1087 & 178 & 35 \\
\hline $\begin{array}{l}\text { Coverage and connectiv- } \\
\text { ity/Optimal deployment }\end{array}$ & 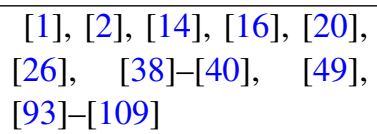 & 36034 & 16346 & 59 \\
\hline $\begin{array}{l}\text { Data gathering/Network } \\
\text { coding }\end{array}$ & $\begin{array}{l}\text { [23], [24], [105], [110]- } \\
\text { [117] }\end{array}$ & 2288 & 779 & 59 \\
\hline Data correlation & [53], [78], [110], [118] & 294 & 165 & 35 \\
\hline Energy harvesting & [14], [109], [119]-[125] & 100 & 22 & 10 \\
\hline Beamforming & {$[30],[126],[127]$} & 50 & 34 & 5 \\
\hline
\end{tabular}


TABLE III

OBJECTIVE FUNCTION(S) (OF), CONSTRAINT(S) AND OPTIMIZATION ALGORITHM(S) IN THE CONTEXT OF RESOURCE ALLOCATION TECHNIQUES THAT MAXIMIZE THE LIFETIME OF WSNS.

\begin{tabular}{|c|c|c|c|c|}
\hline Year & Author(s) & $\mathbf{O F}(\mathbf{s})$ & Constraint Function(s) & Optimization tool(s) and optimality \\
\hline 2004 & $\begin{array}{l}\text { Hoesel et } \\
\text { al. [56] }\end{array}$ & NL-2: NL & $\begin{array}{l}\text { Const.-1,-2: Time-division multiple } \\
\text { access (TDMA)-based MAC proto- } \\
\text { col, sleep scheduling, routing, dy- } \\
\text { namic topology }\end{array}$ & $\begin{array}{l}\text { An on-demand source routing algorithm [129] } \\
\text { using OMNeT++. Optimal solution is obtained. }\end{array}$ \\
\hline \multirow[t]{3}{*}{2006} & $\begin{array}{l}\text { Kwon et } \\
\text { al. [47] }\end{array}$ & NL-1: NL & $\begin{array}{l}\text { Const.-1,-2: Power control, retrans- } \\
\text { mission control, energy efficient } \\
\text { routing, end-to-end transmission } \\
\text { success probability }\end{array}$ & $\begin{array}{l}\text { Greedy power allocation, cost-based routing, } \\
\text { greedy retry limit allocation, cost-based rout- } \\
\text { ing and power control algorithms [47]. Low- } \\
\text { complexity suboptimal solution and optimal so- } \\
\text { lution at the expense of high-complexity are ob- } \\
\text { tained. }\end{array}$ \\
\hline & $\begin{array}{l}\text { Madan et } \\
\text { al. [9] }\end{array}$ & NL-1: NL & $\begin{array}{l}\text { Const.-2,-5: Flow conservation, rate } \\
\text { constraints, energy conservation, } \\
\text { power limits, link scheduling }\end{array}$ & $\begin{array}{l}\text { An iterative algorithm solving a series of convex } \\
\text { optimization problems. Suboptimal solution is ob- } \\
\text { tained. }\end{array}$ \\
\hline & $\begin{array}{l}\text { Nama et } \\
\text { al. [57] }\end{array}$ & $\begin{array}{l}\text { NL-1: NL, } \\
\text { application } \\
\text { perfor- } \\
\text { mance }\end{array}$ & $\begin{array}{l}\text { Const.-2,-5: Source rate control, re- } \\
\text { source allocation, flow control, ED } \\
\text { constraint }\end{array}$ & $\begin{array}{l}\text { An iterative algorithm based on subgradient } \\
\text { method [130]. Optimal solution is obtained. }\end{array}$ \\
\hline \multirow[t]{2}{*}{2007} & $\begin{array}{l}\text { Madan et } \\
\text { al. [58] }\end{array}$ & NL-1: NL & $\begin{array}{l}\text { Const.-2,-5: Rate and power alloca- } \\
\text { tion, flow and energy conservation, } \\
\text { scheduling }\end{array}$ & $\begin{array}{l}\text { An iterative algorithm for finding the optimal } \\
\text { transmission scheme. Suboptimal solution is ob- } \\
\text { tained. }\end{array}$ \\
\hline & $\begin{array}{l}\text { Zhu et al. } \\
\text { [59] }\end{array}$ & $\begin{array}{l}\text { NL-1: NL, } \\
\text { fair rate al- } \\
\text { location }\end{array}$ & $\begin{array}{l}\text { Const.-1,-2: Flow constraints, } \\
\text { power control, energy constraints, } \\
\text { MAC contention }\end{array}$ & $\begin{array}{l}\text { A fully distributed algorithm considering network } \\
\text { utility maximization framework. Suboptimal solu- } \\
\text { tion is obtained. }\end{array}$ \\
\hline 2008 & $\begin{array}{l}\mathrm{Li} \text { et al. } \\
{[60]}\end{array}$ & NL-1: NL & $\begin{array}{l}\text { Const.-2,-5: Rate and power allo- } \\
\text { cation, capacity limits, scheduling, } \\
\text { ED, rate distortion, delay constraint }\end{array}$ & $\begin{array}{l}\text { Successive convex } \\
\text { algorithm [131]. Optimal solution for TDMA, } \\
\text { suboptimal solution for non-orthogonal multiple } \\
\text { access (NOMA) are obtained. }\end{array}$ \\
\hline 2009 & $\begin{array}{l}\text { Phan et } \\
\text { al. [38] }\end{array}$ & NL-1: NL & $\begin{array}{l}\text { Const.-2,-6: Sensor node admission } \\
\text { and deployment, power allocation, } \\
\text { link scheduling }\end{array}$ & $\begin{array}{l}\text { Cross-layer optimization framework based on } \\
\text { mixed integer linear programming using CPLEX } \\
\text { library [132]. Optimal solution is obtained. }\end{array}$ \\
\hline 2011 & $\begin{array}{l}\text { Luo et al. } \\
{[61]}\end{array}$ & $\begin{array}{l}\text { NL-1: NL, } \\
\text { throughput }\end{array}$ & 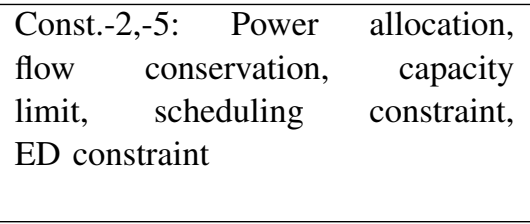 & $\begin{array}{l}\text { Algorithms for max-min NL with max-min } \\
\text { throughput, for maximizing the throughput under } \\
\text { NL constraint, for maximizing the NL under } \\
\text { throughput constraint. Optimal solution is ob- } \\
\text { tained. }\end{array}$ \\
\hline 2012 & $\begin{array}{l}\text { Ehsan et } \\
\text { al. [62] }\end{array}$ & NL-1: NL & $\begin{array}{l}\text { Const.-1,-2,-5: MAC contention } \\
\text { control, rate requirement, ED } \\
\text { constraint, flow balance constraint }\end{array}$ & $\begin{array}{l}\text { Routing schemes based on linear programming } \\
\text { models and mixed integer programming model } \\
\text { using CPLEX [132] and Matlab. Suboptimal so- } \\
\text { lution is obtained. }\end{array}$ \\
\hline 2013 & $\begin{array}{l}\text { Jeon et al. } \\
\text { [63] }\end{array}$ & NL-1: NL & $\begin{array}{l}\text { Const.-2,-5: Contention and sleep } \\
\text { control probability, throughput and } \\
\text { SINR requirements, energy con- } \\
\text { straints }\end{array}$ & $\begin{array}{l}\text { An algorithm based on subgradient method [130] } \\
\text { for finding the optimal Lagrange multipliers. Op- } \\
\text { timal solution is obtained. }\end{array}$ \\
\hline 2014 & $\begin{array}{l}\text { Xu et al. } \\
\text { [37] }\end{array}$ & $\begin{array}{l}\text { NL-1: NL, } \\
\text { rate, relia- } \\
\text { bility }\end{array}$ & $\begin{array}{l}\text { Const.-2,-4,-5: Capacity limits, reli- } \\
\text { ability and ED constraints }\end{array}$ & $\begin{array}{l}\text { Stochastic subgradient algorithm [133], [134]. } \\
\text { Optimal solution is obtained. }\end{array}$ \\
\hline
\end{tabular}




$$
\begin{array}{cl}
\operatorname{min.} & z \\
\text { s.t. } & \mathbf{A}\left(\mathbf{r}_{1}+\mathbf{r}_{2}+\ldots+\mathbf{r}_{N}\right)=\mathbf{s} \cdot N, \\
& \left(\frac{N_{0}}{G_{i, j}} e^{r_{l_{i, j}, n}-Q_{l_{i, j}, n}}+\sum_{i^{\prime} \neq i, l_{i^{\prime}, j^{\prime}} \in \mathcal{L}_{n}} \frac{G_{i^{\prime}, j}}{G_{i, j}} e^{\left.r_{l_{i, j}, n}+Q_{l_{i^{\prime}, j^{\prime}, n}-Q_{l_{i, j}, n}}\right)}-1 \leq 0, \forall n, l, l \in \mathcal{L}_{n},\right. \\
& \sum_{n=1}^{N}\left(\sum_{l \in \mathcal{O}(i) \cap \mathcal{L}_{n}}\left((1+\alpha) \cdot e^{Q_{l_{i, j}, n}}+P_{c t}\right)+\sum_{l \in \mathcal{I}(i) \cap \mathcal{L}_{n}} P_{c r}\right) \leq z \cdot \mathcal{E}_{i} \cdot N, \forall i, \\
& Q_{l_{i, j}, n} \leq \log \left(\left(P_{i}\right)_{\max }\right), l \in \mathcal{L}_{n}, \\
& \mathbf{r}_{n} \geq 0, \forall n, \\
& r_{l_{i, j}, n}=0, \forall l \notin \mathcal{L}_{n} .
\end{array}
$$

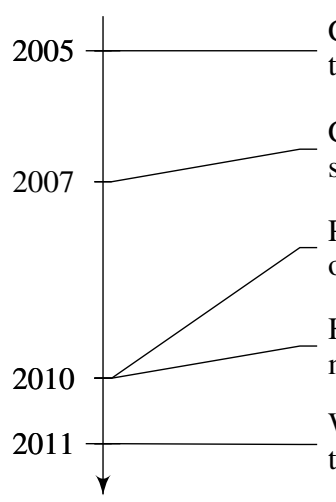

Chen et al. [8] advocated an efficient MAC protocol, which relies both on the channel state information and on the MAC's knowledge of the residual energy in order to maximize the NL.

Chen et al. [67] focused their attention on the transmission scheduling relying on the opportunistic channel state information for the sake of NL maximization.

Phan et al. [68] proposed an energy-efficient transmission scheme based on the prevalent channel conditions in order to maximize the NL.

Hung et al. [69] developed a routing protocol exploiting the advantages of opportunistic routing in order to maximize the NL.

Wu et al. [70] proposed a coalition formation game-theory method in the interest of selecting the best possible transmission scheme for maximizing the NL.

Fig. 6. Timeline of opportunistic transmission techniques that maximize the lifetime of WSNs.

$$
\begin{aligned}
Q_{l_{i, j}, n}^{t+1}= & \log \left[( \mu _ { i } ^ { t } - \mu _ { j } ^ { t } - \vartheta _ { l _ { i , j } , n } ^ { t } ) \cdot \left(\omega_{i}^{t}(1+\alpha)+\sum_{l_{i^{\prime}, j^{\prime}} \in \mathcal{L}_{n}, l_{i^{\prime}, j^{\prime}} \neq l_{i, j}, i^{\prime} \geq i} \psi_{l_{i^{\prime}, j^{\prime}}, n}^{t}\left(\frac{G_{i, j^{\prime}}}{G_{i^{\prime}, j^{\prime}}} \cdot e^{r_{l_{i^{\prime}, j^{\prime}, n}}^{t}-Q_{l_{i^{\prime}, j^{\prime}, n}}^{t}}\right)\right.\right. \\
& \left.\left.+\sum_{l_{i^{\prime}, j^{\prime}} \in \mathcal{L}_{n}, l_{i^{\prime}, j^{\prime}} \neq l_{i, j}, i^{\prime}<i} \psi_{l_{i^{\prime}, j^{\prime}}, n}^{t}\left(\frac{G_{i, j^{\prime}}}{G_{i^{\prime}, j^{\prime}}} \cdot e^{r_{l_{i^{\prime}, j^{\prime}, n}}^{t}-Q_{l_{i^{\prime}, j^{\prime}}, n}^{t+1}}\right)\right)^{-1}\right], \forall l, n, \\
r_{l_{i, j}, n}^{t+1}= & \log \left[\frac{\mu_{i}^{t}-\mu_{j}^{t}}{\psi_{l_{i, j}, n}^{t} \cdot\left(\frac{N_{0}}{G_{i, j}}+\sum_{l_{i^{\prime}, j^{\prime}} \in \mathcal{L}_{n}, l_{i^{\prime}, j^{\prime}} \neq l_{i, j}} \frac{G_{i^{\prime}, j}}{G_{i, j}} \cdot e^{\left.Q_{l_{i^{\prime}, j^{\prime}, n}}^{t+1}\right)}\right]+Q_{l_{i, j}, n}^{t+1}, \forall l, n .}\right.
\end{aligned}
$$

their optimization algorithms and optimality are surveyed in Table III, which also summarizes the particular resources allocated in the $O F(s)$ and Constraint Function(s) columns.

\section{B. Opportunistic Transmission Schemes and Sleep-Wake Scheduling}

Once the information has been gathered by the sensors, its transmission to the sink node can be initiated. However, it has to be carefully considered, which specific group of sensors should relay the sensed data to the DN, at which instant in time, especially when communicating over fading channels. Plausible logic dictates that transmission using those particular sensors, which momentarily experience better chan- nels conserves considerable amount of energy. Matamoros et al. [66] proposed opportunistic power allocation and sensor selection schemes for parameter estimation, where only the specific sensors enjoying favorable channel conditions were involved in the estimation of the data transmitted via adapting their transmit power relying on both the channel state information and the residual battery charge information in order to enhance the NL. Furthermore, Chen and Zhao [8] advocated an efficient MAC protocol, which relies both on the channel state information and on the MAC's knowledge of the residual energy in order to maximize the NL. In [67] the authors focused their attention on the transmission scheduling of specific access points communicating over a fading channel 


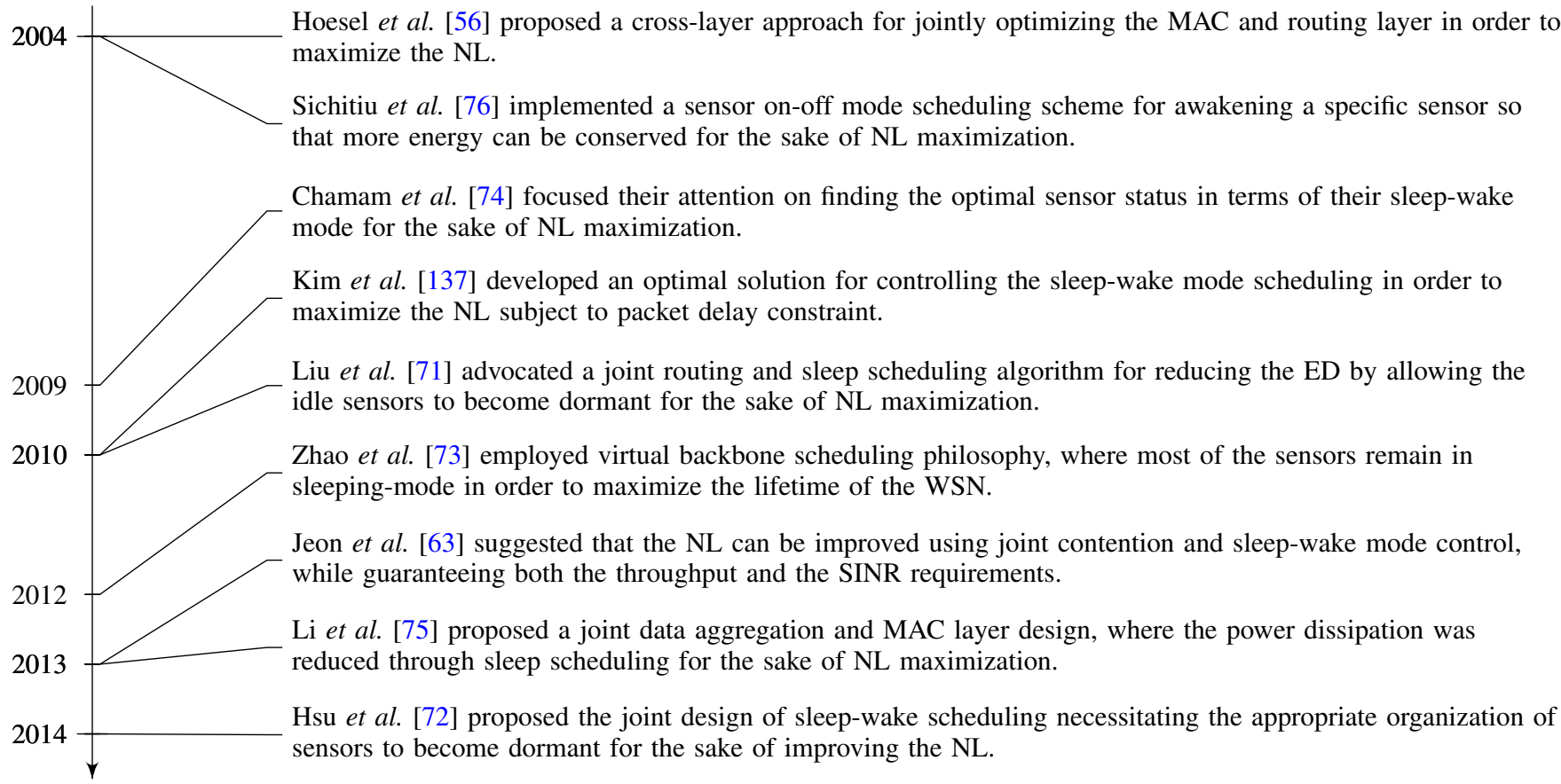

Fig. 7. Timeline of the sleep scheduling techniques that maximize the NL.

relying both on the opportunistic channel state information and on the remaining battery charge information for the sake of NL maximization. Phan et al. [68] proposed an energy-efficient transmission scheme based on the prevalent channel conditions in order to maximize the NL. More explicitly, transmissions were only activated, when the channel quality was above a predefined threshold, while communicating over fading channels. Moreover, a routing protocol exploiting the advantages of opportunistic routing in order to maximize the NL was presented in [69], where both the end-to-end transmission cost as well as the residual battery charge of each sensor and the transmission success probability of each relay node was jointly considered. As a further development, $\mathrm{Wu}$ et al. [70] proposed a coalition formation game-theory framework in the interest of selecting the best possible transmission scheme for maximizing the NL. In this section, the major contributions on the subject of the opportunistic transmission techniques conceived in the interest of maximizing the NL are summarized in Fig. 6, whilst their OFs, constraints, optimization algorithms and optimality are surveyed in Table IV.

Nonetheless, the employment of sleep-wake mode based scheduling can be extremely beneficial in terms of an extended $\mathrm{NL}$, especially in application scenarios, when the packets only arrive sporadically. Hence, Kim et al. [137] developed an optimal solution for controlling the sleep-wake mode scheduling of a so-called anycast packet-forwarding scheme ${ }^{2}$ in order to maximize the NL subject to packet delay constraints. Another example of the sleep-wake mode scheduling can be found in [71], where Liu et al. advocated a joint routing and sleep scheduling algorithm for balancing the tele-traffic load across

\footnotetext{
${ }^{2}$ Each sensor node opportunistically transmits a packet to the closest neighboring sensor node that wakes up in the set of multiple sensor nodes.
}

the entire network and for reducing the ED by allowing the idle sensors to become dormant. The algorithm proposed in [71] extended the NL by $29 \%$ compared to either an optimal routing scheme dispensing with sleep-wake scheduling or compared to a pure sleep scheduling scheme. As a benefit, a NL improvement of about $284 \%$ was observed compared to the conventional optimal routing schemes relying on a fixed sleep scheduling. Similarly, Hsu et al. [72] proposed the joint design of sleep-wake scheduling necessitating the appropriate organization of sensors to become dormant for the sake of improving the energy-efficiency and of opportunistic routing, which improved the routing diversity by spatially distributing the tele-traffic. This improved the reliability of transmission across the network, whilst additionally improving the NL.

An interesting sleep scheduling approach, namely the virtual backbone scheduling philosophy was employed in [73], where the traffic is only forwarded through the so-called backbone sensor nodes constituted by the non-correlated sensor nodes, while the rest of the sensors remain in sleeping-mode in the WSN considered. The sleep scheduling approach of Zhao et al. [73] provided a spatially balanced distribution of the ED and thus maximized the NL. Jeon et al. [63] suggested that the NL can be improved using joint contention and sleep-wake mode control, while guaranteeing both the throughput and the SINR requirements. Furthermore, Chamam et al. [74] focused their attention on finding the optimal sensor status in terms of their sleep-wake mode as well as their potential cluster head status for the sake of NL maximization subject to coverage, clustering and routing constraints. A joint data aggregation and MAC layer design was proposed by Li et al. [75], where both the network traffic was carefully adjusted with the aid of data aggregation and the power dissipation was reduced 
TABLE IV

OF(S), CONSTRAINT(S) AND OPTIMIZATION ALGORITHM(S) IN THE CONTEXT OF OPPORTUNISTIC TRANSMISSION TECHNIQUES THAT MAXIMIZE THE LIFETIME OF WSNS.

\begin{tabular}{|c|c|c|c|c|}
\hline Year & Author(s) & $\mathbf{O F}(\mathbf{s})$ & Constraint function(s) & Optimization tool(s) and optimality \\
\hline 2005 & Chen et al. [8] & NL-1,-12: NL & $\begin{array}{l}\text { Const.-1,-2: Channel state informa- } \\
\text { tion (CSI), residual energy informa- } \\
\text { tion (REI) }\end{array}$ & $\begin{array}{l}\text { A greedy max-min algorithm [138] in } \\
\text { order to maximize the NL by exploit- } \\
\text { ing CSI and REI. Optimal solution is } \\
\text { obtained. }\end{array}$ \\
\hline 2007 & $\begin{array}{l}\text { Chen et al. } \\
\text { [67] }\end{array}$ & NL-12: NL & $\begin{array}{l}\text { Const.-1,-2: Transmission schedul- } \\
\text { ing, CSI, REI, optimal scheduling }\end{array}$ & $\begin{array}{l}\text { Formulated as a stochastic shortest path } \\
\text { Markov decision process [139] and } \\
\text { solved using a dynamic protocol for life- } \\
\text { time maximization (DPLM), which pro- } \\
\text { vides suboptimal solution. }\end{array}$ \\
\hline \multirow[t]{2}{*}{2010} & Phan et al. [68] & NL-1: NL & $\begin{array}{l}\text { Const.-1,-2: Instantaneous channel } \\
\text { conditions, energy efficient trans- } \\
\text { mission scheme, throughput, end- } \\
\text { to-end delay }\end{array}$ & $\begin{array}{l}\text { An algorithm based on binary decision } \\
\text { aided transmission with channel aware } \\
\text { back-off adjustment. Suboptimal solu- } \\
\text { tion is obtained. }\end{array}$ \\
\hline & $\begin{array}{l}\text { Hung et al. } \\
\text { [69] }\end{array}$ & NL-9: NL & $\begin{array}{l}\text { Const.-2,-4: Opportunistic routing, } \\
\text { path diversity, reliability, delay }\end{array}$ & $\begin{array}{l}\text { A distributed routing scheme, namely } \\
\text { the so-called energy-efficient opportunis- } \\
\text { tic routing technology (EFFORT) [140], } \\
\text { which provides optimal solution. }\end{array}$ \\
\hline 2011 & Wu et al. [70] & NL-1,-12: NL & $\begin{array}{l}\text { Const.-1,-2: Transmission scheme } \\
\text { selection, transmission distance, } \\
\text { outage probability, power allocation }\end{array}$ & $\begin{array}{l}\text { A coalition formation game [141] us- } \\
\text { ing nontransferable utility game theory } \\
\text { model. A stronger stability solution can } \\
\text { be achieved, which leads to the global } \\
\text { optimum. }\end{array}$ \\
\hline
\end{tabular}

through sleep scheduling, which were jointly considered under the constraint of a specific packet delivery delay.

Hoesel et al. [56] proposed a cross-layer approach for jointly optimizing the MAC and routing layer in order to maximize the NL, where the MAC layer is in charge of setting the sensors to their active or inactive mode, while the routing layer identifies efficient routes in the face of a dynamic node topology. Finally, an energy conservation method was designed by Sichitiu et al. [76] for the sake of NL maximization, where a sensor on-off mode scheduling scheme was proposed for awakening a specific sensor, if and only if necessary. In this section, the major contributions on the subject of sleep-wake-up mode scheduling techniques maximizing the NL are summarized in Fig. 7, while their OFs, constraints, optimization algorithms and optimality are surveyed in Table V.

\section{Routing and Clustering}

Routing decisions play a significant role in determining the achievable NL. Specifically, constructing lifetime-aware routes is crucial for the sake of NL maximization, since a dynamic route created by the sensors having the maximum residual battery charge can be beneficially exploited, each time when a transmission from the SN to DN is initiated, which assists the network in balancing the overall ED and ultimately in extending its lifetime. In order to maximize the $\mathrm{NL}$, in [77] the routing of the tele-traffic had to be balanced across the WSN considered, since repeatedly using the same route depletes the battery of the corresponding sensors more rapidly than that of the rest of the sensors and thus degrades the NL. However, exploiting the battery energy of the remaining active sensors has the potential of extending the NL. Therefore, optimizing the routes directly affects the NL. For instance, Liu et al. [71] considered a joint routing and sleep-mode scheduling algorithm for balancing the traffic load across the entire network and for reducing the ED by allowing the idle sensors to switch to their sleep mode. The joint optimization based algorithm proposed in [71] extended the NL by $29 \%$ compared to either the pure routing optimized scheme or to a pure sleep-mode scheduling scheme operating without their joint optimization. Furthermore, a dramatic NL improvement of about $284 \%$ was observed compared to the conventional optimal routing schemes relying on a fixed sleepmode scheduling. Similarly, Hsu et al. [72] proposed the appropriate organization of sensors for jointly optimizing both their sleep-mode for energy-efficiency and their opportunistic routing for the sake of balancing their traffic load distribution and for improving the attainable transmission reliability across the network for the sake of NL maximization. On the other 
TABLE V

OF(s), CONSTRAINT(S) AND OPTIMIZATION ALGORITHM(S) IN THE CONTEXT OF SLEEP-WAKE SCHEDULING TECHNIQUES THAT MAXIMIZE THE LIFETIME OF WSNS.

\begin{tabular}{|c|c|c|c|c|}
\hline Year & Author(s) & OF(s) & Constraint function(s) & Optimization tool(s) and optimality \\
\hline \multirow[t]{2}{*}{2004} & $\begin{array}{l}\text { Hoesel et } \\
\text { al. [56] }\end{array}$ & NL-2: NL & $\begin{array}{lr}\text { Const.-1,-2: } r & \text { TDMA-based } \\
\text { MAC protocol, } & \text { sleep } \\
\text { scheduling, routing, dynamic } \\
\text { topology }\end{array}$ & $\begin{array}{l}\text { An on-demand source routing algorithm [129] using } \\
\text { OMNeT++. Optimal solution is obtained. }\end{array}$ \\
\hline & $\begin{array}{l}\text { Sichitiu et } \\
\text { al. [76] }\end{array}$ & NL-2: NL & $\begin{array}{l}\text { Const.-1,-2,-4: Sleep schedul- } \\
\text { ing, energy conservation }\end{array}$ & $\begin{array}{l}\text { A distributed sleep-awake based scheduling algorithm } \\
\text { relying on energy conservation scheme. Optimal so- } \\
\text { lution is obtained. }\end{array}$ \\
\hline 2009 & $\begin{array}{l}\text { Chamam } \\
\text { et al. [74] }\end{array}$ & NL-8: NL & $\begin{array}{lrr}\text { Const.-2,-3: } & \text { Coverage } & \text { quality, } \\
\text { clustering, } & \text { routing, } & \text { sleep } \\
\text { scheduling } & & \end{array}$ & $\begin{array}{l}\text { TABU search heuristic algorithm [142] providing a } \\
\text { suboptimal solution with reduced complexity and } \\
\text { an integer linear programming model providing an } \\
\text { optimal solution at the cost of high-complexity, which } \\
\text { is solved using CPLEX library [132]. }\end{array}$ \\
\hline \multirow{2}{*}{2010} & $\begin{array}{l}\text { Kim et } \\
\text { al. [137] }\end{array}$ & NL-1: NL & $\begin{array}{l}\text { Const.-1,-2: } \quad \text { Sleep-wake } \\
\text { scheduling, minimizing packet } \\
\text { delay, any-cast forwarding }\end{array}$ & $\begin{array}{l}\text { An optimal any-cast algorithm based on the value- } \\
\text { iteration and local optimal algorithms. }\end{array}$ \\
\hline & $\begin{array}{l}\text { Liu et } \\
\text { al. [71] }\end{array}$ & NL-1: NL & $\begin{array}{l}\text { Const.-1,-2: Balanced traffic } \\
\text { routing, sleep scheduling }\end{array}$ & $\begin{array}{l}\text { An iterative geometric programming algorithm [131] } \\
\text { based on signomial programming [143] problem. } \\
\text { Near-optimal solution is obtained. }\end{array}$ \\
\hline 2012 & $\begin{array}{l}\text { Zhao et } \\
\text { al. [73] }\end{array}$ & NL-1: NL & $\begin{array}{l}\text { Const.-1,-2: Sleep scheduling, } \\
\text { energy-delay trade-off, virtual } \\
\text { backbone scheduling }\end{array}$ & $\begin{array}{l}\text { Schedule transition graph, virtual scheduling graph } \\
\text { algorithms, distributed iterative local replacement } \\
\text { scheme. Suboptimal solutions are obtained. }\end{array}$ \\
\hline \multirow[t]{2}{*}{2013} & $\begin{array}{l}\text { Jeon et } \\
\text { al. }[63]\end{array}$ & NL-1: NL & $\begin{array}{l}\text { Const.-1,-2,-5: } \text { Contention and } \\
\text { sleep control probability, } \\
\text { throughput and } \begin{array}{r}\text { SINR } \\
\text { requirements, } \\
\text { constraints }\end{array}\end{array}$ & $\begin{array}{l}\text { An algorithm based on subgradient method [130] for } \\
\text { finding the optimal Lagrange multipliers [136]. }\end{array}$ \\
\hline & $\begin{array}{l}\mathrm{Li} e t \\
\text { al. }[75]\end{array}$ & NL-1: NL & $\begin{array}{l}\text { Const.-1,-2: Data aggregation, } \\
\text { reduced network traffic, sleep } \\
\text { scheduling, packet delivery de- } \\
\text { lay }\end{array}$ & $\begin{array}{l}\text { Joint aggregation and MAC holistic approach using } \\
\text { NS- } 2 \text { simulations and testbed experiments. Subopti- } \\
\text { mal solution is obtained. }\end{array}$ \\
\hline 2014 & $\begin{array}{l}\text { Hsu et } \\
\text { al. [72] }\end{array}$ & NL-1: NL & $\begin{array}{l}\text { Const.-1,-2: Sleep scheduling, } \\
\text { traffic balance, route diversity, } \\
\text { transmission reliability, oppor- } \\
\text { tunistic routing }\end{array}$ & $\begin{array}{l}\text { Joint design of asynchronous sleep-wake scheduling } \\
\text { and opportunistic routing technology. Suboptimal so- } \\
\text { lution is obtained. }\end{array}$ \\
\hline
\end{tabular}

hand, the joint optimization of the data aggregation ${ }^{3}$ and maximum-lifetime-oriented routing was considered in [78], where the data aggregation reduces the traffic-load across the network by avoiding the transmission of the redundant data, which is identified with the aid of the temporal-spatial data correlation. Hence, the power dissipation of the sensor nodes that are adjacent to the sink node can be substantially reduced, while the maximum-lifetime routing policy balances

\footnotetext{
${ }^{3}$ Data aggregation is an information processing technique, which incorporates data arriving from various sensor nodes in order to cope with the spatial and temporal data correlation by eliminating the redundant information, while minimizing the number of transmissions with the aid of aggregators located at specific sensor nodes.
}

the traffic for avoiding the overloading some of the sensors. Additionally, Amiri et al. [79] studied the joint optimization of traffic routing and camera selection strategy for the sake of NL maximization, where efficient sensor collaboration was required for data sensing and camera selection for the sake of extending the NL. This approach supports the collaboration of different sensors to avoid redundant sensing of various areas in the WSN and assists in the cooperative routing of the teletraffic generated. In [80], Al-Shawi et al. developed a routing algorithm for WSNs for extending the NL, where the aim is to find an optimal route from the SN to the sink node with the aid of the highest remaining battery charge, the minimum number 
TABLE VI

OF(s), CONSTRAINT(S) AND OPTIMIZATION ALGORITHM(S) IN THE CONTEXT OF ROUTING TECHNIQUES THAT MAXIMIZE THE LIFETIME OF WSNS.

\begin{tabular}{|c|c|c|c|c|}
\hline Year & Author(s) & OF(s) & Constraint function(s) & Optimization tool(s) and optimality \\
\hline 2000 & $\begin{array}{lll}\text { Chang } & \text { et } & \text { al. } \\
\text { [77] } & & \end{array}$ & NL-1: NL & $\begin{array}{lccc}\text { Const.-2: } & \text { Balancing } & \text { ED } & \text { rates } \\
\text { amongst nodes, flow routing } & \end{array}$ & $\begin{array}{l}\text { Flow augmentation algorithm [144], flow } \\
\text { redirection algorithm [145]. Suboptimal } \\
\text { solution is obtained. }\end{array}$ \\
\hline 2004 & $\begin{array}{l}\text { Chang et al. } \\
\text { [82] }\end{array}$ & NL-1: NL & $\begin{array}{l}\text { Const.-2: Flow conservation, rout- } \\
\text { ing control packets, ED rate, resid- } \\
\text { ual energy levels }\end{array}$ & $\begin{array}{l}\text { Flow augmentation algorithm based on the } \\
\text { shortest path routing strategy using the } \\
\text { link cost quantified by the communica- } \\
\text { tion ED and residual energy levels. Near- } \\
\text { optimal solution is obtained. }\end{array}$ \\
\hline 2008 & Hua et al. [78] & NL-1: NL & $\begin{array}{l}\text { Const.-2: Traffic reduction, traffic } \\
\text { balancing, data aggregation }\end{array}$ & $\begin{array}{l}\text { Maximum lifetime routing algorithm us- } \\
\text { ing routing adaptation and the classic } \\
\text { gradient method. Optimal solution is ob- } \\
\text { tained. }\end{array}$ \\
\hline \multirow{3}{*}{2009} & $\begin{array}{l}\text { Cheng et al. } \\
{[43]}\end{array}$ & NL-1: NL & $\begin{array}{l}\text { Const.-2: Energy and bandwidth } \\
\text { constraints, link rate allocation, } \\
\text { routing }\end{array}$ & $\begin{array}{l}\text { Algorithms for scalable rate allocation } \\
\text { along the shortest paths and optimizing } \\
\text { the lifetime subject to a bandwidth con- } \\
\text { straint. Suboptimal solution is obtained. }\end{array}$ \\
\hline & Li et al. [28] & NL-11: NL & $\begin{array}{l}\text { Const.-2: Source coding, source } \\
\text { throughput, multihop routing }\end{array}$ & $\begin{array}{l}\text { An algorithm using character-based rout- } \\
\text { ing [28], which relays data only over } \\
\text { nodes having higher importance. Optimal } \\
\text { solution is obtained. }\end{array}$ \\
\hline & He et al. [83] & NL-1: NL & $\begin{array}{l}\text { Const.-2: Source rate, encoding } \\
\text { power, routing scheme }\end{array}$ & $\begin{array}{l}\text { A distributed algorithm using subgradient } \\
\text { method [130], [146]. Optimal solution is } \\
\text { obtained. }\end{array}$ \\
\hline 2010 & Liu et al. [71] & NL-1: NL & $\begin{array}{l}\text { Const.-1,-2: Balanced traffic rout- } \\
\text { ing, sleep scheduling }\end{array}$ & $\begin{array}{l}\text { An iterative geometric programming al- } \\
\text { gorithm [131] based on signomial pro- } \\
\text { gramming [143] problem. Near-optimal } \\
\text { solution is obtained. }\end{array}$ \\
\hline 2011 & $\begin{array}{l}\text { Amiri et al. } \\
\text { [79] }\end{array}$ & NL-1: NL & $\begin{array}{l}\text { Const.-2: Traffic routing, camera } \\
\text { selection strategy, node collabora- } \\
\text { tion }\end{array}$ & $\begin{array}{l}\text { An optimal collaborative routing and cam- } \\
\text { era selection algorithm, a low-complexity } \\
\text { suboptimal heuristic routing and camera } \\
\text { selection algorithm. }\end{array}$ \\
\hline 2012 & $\begin{array}{l}\text { Al-Shawi et al. } \\
\text { [80] }\end{array}$ & NL-1: NL & $\begin{array}{l}\text { Const.-2: Optimal route, residual } \\
\text { battery charge, number of hops, } \\
\text { traffic load }\end{array}$ & $\begin{array}{l}\text { An optimal path algorithm based on the } \\
\text { joint design of a fuzzy approach [147] and } \\
\text { an A-star algorithm [148]. }\end{array}$ \\
\hline 2013 & Peng et al. [81] & NL-1: NL & $\begin{array}{l}\text { Const.-1,-2: Balancing node life- } \\
\text { time, delivery delay constraint, } \\
\text { power dissipation }\end{array}$ & $\begin{array}{l}\text { A holistic lifetime balancing technique, } \\
\text { namely the so-called intra-route and inter- } \\
\text { route coordination method. }\end{array}$ \\
\hline \multirow{2}{*}{2014} & $\begin{array}{l}\text { Cassandras et } \\
\text { al. [12] }\end{array}$ & NL-1: NL & $\begin{array}{lcr}\text { Const.-1,-2: } & \text { Optimal } & \text { routing } \\
\text { scheme, } & \text { nonlinear } & \text { battery } \\
\text { discharging, balancing ED } & \end{array}$ & $\begin{array}{l}\text { An algorithm solving a set of simpler } \\
\text { non-linear programming problems based } \\
\text { on kinetic battery model [149]. Optimal } \\
\text { solution is obtained. }\end{array}$ \\
\hline & Hsu et al. [72] & NL-1: NL & $\begin{array}{l}\text { Const.-1,-2: Sleep scheduling, traf- } \\
\text { fic balance, route diversity, trans- } \\
\text { mission reliability, opportunistic } \\
\text { routing }\end{array}$ & $\begin{array}{l}\text { Joint design of asynchronous sleep-wake } \\
\text { scheduling and opportunistic routing tech- } \\
\text { nology. Suboptimal solution is obtained. }\end{array}$ \\
\hline
\end{tabular}




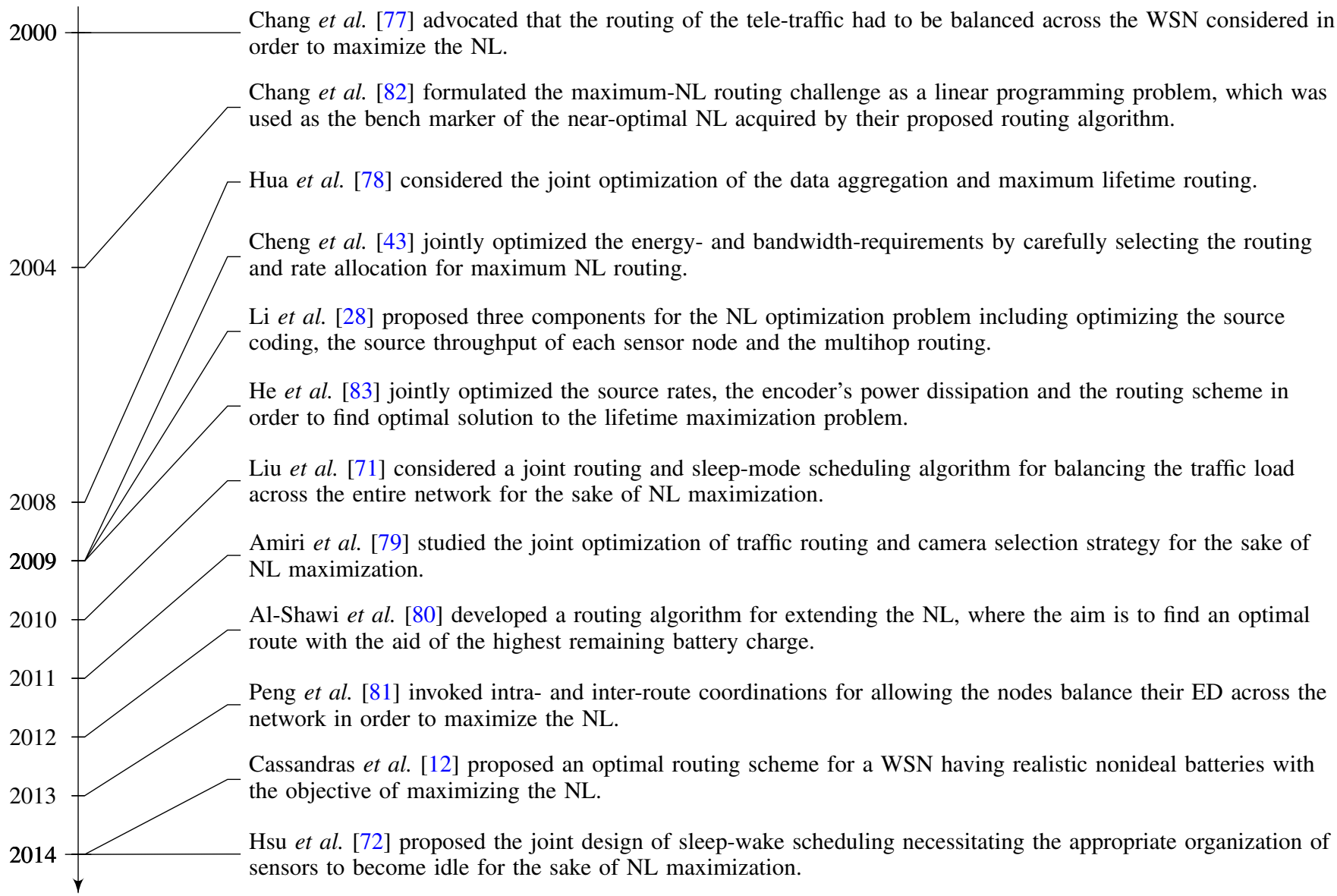

Fig. 8. Timeline of the routing optimization techniques that maximize the NL.

of hops and the minimum traffic load. Peng et al. [81] invoked intra-route coordination for allowing the nodes along the same route to balance their node lifetime durations, which was also combined with inter-route coordination for additionally balancing the lifetime durations of the sensors along different routes in order to collaboratively maximize the NL. This was carried out under a specific delivery delay constraint.

A cross-layer approach conceived for maximizing the NL was proposed in [62], where MAC-aware routing optimization schemes were designed for WSNs that are capable of multichannel access. Another cross-layer approach was conceived for maximum NL routing in [43], where the energy- and bandwidth-requirements were jointly optimized by carefully selecting the routing and rate allocation in a bandwidthand energy-constrained WSN. Additionally, Chang et al. [82] formulated the maximum-NL routing challenge as a linear programming problem, which was used as the benchmarker of the near-optimal NL acquired by their proposed routing algorithm. However, the design goal in [82] was to simply find the specific flow that maximizes the NL relying on the flow conservation constraint ${ }^{4}$. Additionally, an optimal routing scheme was proposed in [12] with the objective of maximizing the NL, where the authors considered realistic nonideal batteries by modeling the nonlinear ED behavior of the typical batteries.

Li et al. [28] proposed three components for the NL optimization problem including optimizing the source coding, the source throughput of each sensor node and the multihop routing, where the bandwidth-efficient local quantization of the source-information and the employment of energy-conscious multihop routing are widely known to be essential for achieving energy conservation. These three components were formulated as a linear programming problem for maximizing the NL. Similarly, distributed algorithms were developed by $\mathrm{He}$

${ }^{4}$ For any sensor node, flow in is equivalent to flow out. If the source is generating $b_{j}$ flows, in a directed graph with a set $V$ nodes, we have $\sum f_{j, k}-$ $\sum f_{i, j}=b_{j}$, where $f_{i, j}$ represents a flow from SN $i$ to DN $j$, for $i, j \in V$. Explicitly, if only a sensor node is generating source information for a DN, then the intermediate node $k \in V$ can only have the additional amount of flow information that is generated at the $\mathrm{SN}$, so that the flow generated at the $\mathrm{SN}$ is conserved for the $\mathrm{DN}$. 


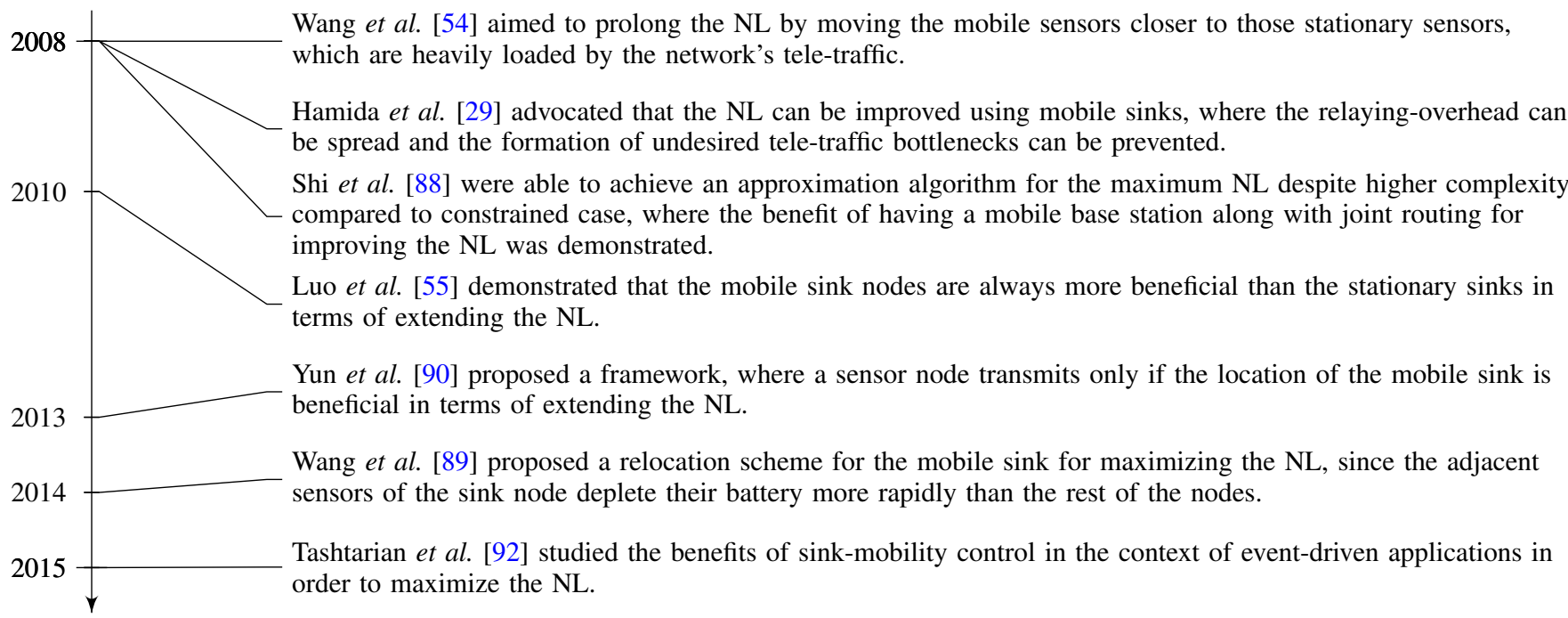

Fig. 9. Timeline of mobility-aided techniques that extend the NL.

et al. [83] by exploiting the so-called Lagrangian duality ${ }^{5}$ in order to find the optimal solution to the lifetime maximization problem, which was formulated based on the joint optimization of the source rates, the encoder's power dissipation and the routing scheme. In this section, the major contributions on the subject of routing optimization techniques designed for NL maximization are surveyed in Fig. 8, while their OFs, constraints, optimization algorithms and optimality are summarized in Table VI.

Nonetheless, long-haul communication with distant areas are costly in terms of ED in battery-powered WSNs. Hence, this scenario necessitates a multi-tier network architecture for relaying the data, while keeping the network operational for the longest possible period of time. An efficient method of increasing the lifetime of WSNs is to partition the network into several clusters under the control of a high-energy cluster head [84], which the network can rely on. Specifically, Gupta et al. [85] proposed an efficient fault-tolerant clustering scheme, where the sensors of a failed cluster may be incorporated into an operational cluster for the sake of network lifetime maximization. Similarly, the same authors developed an algorithm in [86] for exploiting the gateways equipped with a high-energy battery in order to maximize the NL by avoiding spatially unbalanced ED across the network.

\section{Mobile Relays and Sinks}

Data collection at the sink node often results in routingcongestion in the vicinity of sensors neighboring the sink node, since these sensors are frequently used for delivering the data to the DN. This results in rapid battery depletion of these particular sensor nodes and leads to NL reduction

\footnotetext{
${ }^{5} \mathrm{~A}$ minimization problem can be referred to as a primal (original) problem, and there exists a dual maximization problem of that particular minimization primal problem, which can produce the same optimal solution as the primal one. The Lagrangian duality is preferred, since the dual optimization problem is always convex, which can be efficiently solved, even though the primal problem is a nonconvex one.
}

due to the unbalanced traffic-load, thus imposing an unevenly distributed ED across the WSN [87]. A beneficial method of circumventing this problem is to rely on a technique referred to as controlled mobility, which relies on mobile sensors or mobile sinks, where each mobile sensor cooperatively decides its direction of movement in order to prevent an uneven traffic burden distribution. As a benefit, the traffic-load becomes uniformly distributed across the network by taking advantage of the mobility. For instance, in [54] Wang et al. considered a WSN, which is constituted by several mobile relay sensor nodes and a large number of stationary nodes, where the resources of the mobile sensors are richer than those of the stationary sensors. The aim of the work presented in [54] was to prolong the NL by moving the mobile sensors closer to those stationary sensors, which are heavily loaded by the network's tele-traffic. Similarly, Hamida et al. [29] advocated that the NL can be significantly improved via mobile sinks, where the relaying-overhead of sensor nodes that are close to the sink can be spread and the formation of undesired tele-traffic bottlenecks can be prevented. An interesting study on the benefit of having a mobile base station along with joint routing for improving the NL was proposed by Shi et al. [88]. This study considered a constrained location of the base station. Interestingly, Shi et al. [88] demonstrated that when the location of a base station is un-constrained, an approximation algorithm was proposed that has a higher complexity compared to the constrained case, while the NL achieved is close to the maximum NL with a small precision value. Similar to [88], Luo et al. [55] analyzed the effects of joint sink mobility and routing in order to maximize the NL, however they only constrained the position of the mobile sink to a limited number of locations. Consequently, Luo et al. [55] demonstrated that the mobile sink nodes are always more beneficial than the stationary sinks in terms of extending the NL. A relocation scheme was proposed for the mobile sink by Wang et al. [89] for maximizing the NL, since the adjacent sensors of the sink node deplete their battery more 
rapidly than the rest of the nodes in WSNs. Their proposed scheme exploited the knowledge of the remaining battery charge information of the sensor nodes for adaptively adjusting the transmission distance of the sensor nodes and for the beneficial relocation of the sink node.

Additionally, similar studies were carried out in [90], [91], where a sensor node transmits only on condition, if the location of the mobile sink is beneficial in terms of extending the NL, under the additional constraint that each sensor stores its data up to a predetermined delay tolerance threshold. A different approach was proposed by Tashtarian et al. [92], who studied the benefits of sink-mobility control in the context of event-driven applications in order to maximize the NL. More explicitly, in the interest of maximizing the NL, an optimal single-hop link was relied upon in [92] without assuming any specific predetermined network structure, where the mobile sink node has to capture the occurrence of specific events gleaned from a group of sensors, until a certain deadline expired. The main contributions on mobility-aided techniques that extend the NL are reviewed in Fig. 9 of this section, while their OFs, constraints, optimization algorithms and optimality are characterized in Table VII.

\section{E. Coverage, Connectivity and Optimal Deployment}

The term coverage is also referred to as sensing coverage, which indicates the observation quality of specific events within a target area, at a particular sensing point or within a barrier field covered by the sensors deployed. We note that the sensed information is processed relying on a specific hardware component, which is distinct from the transceiver component of the particular sensor device [1], [2]. Naturally, a specific point within the target area may be concurrently sensed by several sensors. While this type of deployment can be beneficial in terms of improving the quality or reliability of the data observed, this also introduces data redundancy, which in turn results in wasted energy. Hence, it is beneficial to critically appraise, whether the data should or should not be transmitted to the base station. Explicitly, if insufficient sensors are deployed, the probability of adequate connectivity to the base station or to another sensor might become inadequately low. Crucially, ensuring high-quality connectivity of these sensors predetermines the ability of transmitting the sensed observations to the base station. As illustrated in Fig. 11, the sensing range $R_{s}$ determines the area within which adequate sensing is achieved, and the transmission range $R_{t}$ defines the area of adequate transmission quality. More explicitly, an observation at points $\mathrm{X}$ and $\mathrm{Y}$ cannot be adequately recorded, since the sensing range of the given sensors is too restricted, even though the points lie within the adequate transmission range. Hence, other sensor nodes have to cover the $\mathrm{X}$ and $\mathrm{Y}$ points, but at the same time the sensors have to remain within the adequate transmission range of node-A and node-B, respectively, so that the observations can be adequately sensed and transmitted to the base station. Regarding this issue, Zhang et al. [93] formally proved that a complete coverage of a convex region implies having adequate connectivity amongst all the sensors deployed, provided that the transmission range is at least twice the sensing range, i.e. we have $R_{t} \geq 2 R_{s}$. Further debates on the subjects of sensing range and transmission range can be found in [20], [93]-[96].

One of the most important constraints of the WSNs is to provide reliable full coverage of a particular sensing field at any moment in time and to relay all the sensed data to the sink node via a subset of the deployed sensors. Chen et al. [97] developed a novel NL maximization algorithm, which allows the activation of the lowest possible number of sensor nodes with the aid of traffic-balancing in order to provide reliable full coverage of a specific sensing field, while providing anytime connectivity to a base station. Similarly, Zhao et al. [26] proposed a scheduling approach necessitating for all the active sensors to maintain full-time coverage of a particular target area all the time and to send all the sensed information to the sink via subsets of sensors, which also requires full-time connectivity to the sink with the aid of multi-hop communication between these subsets. If the coverage of the target area and the anticipated connectivity within the subsets of sensors and the sink node cannot be maintained, then the authors of [26] assumed that the NL expires. Additionally, Deng et al. [98] studied the issues of reliable coverage in the context of agricultural applications of WSNs, assuming that each node is equipped with sensors carrying out different tasks, where the aim was to schedule the activity of these heterogeneous sensors by ensuring that reliable coverage can be maintained, whilst the NL is maximized. As an alternative solution, Lin et al. [99] proposed an ant colony optimization based approach that is capable of maximizing the lifetime of heterogeneous WSNs, where a construction graph is used for determining the maximum number of disjoint connected coverage segments ${ }^{6}$, where each sensor in this disjoint subset can individually maintain both the required coverage quality and reliable network connectivity, while the rest of the sensors of the same disjoint subset are in their sleep-mode. Du et al. [14] also focused their attention on NL maximization subject to the military barrier coverage constraints ${ }^{7}$, where the sensors form continuous geographic area barriers with the goal of detecting the crossing of an area by the adversaries. Additionally, Lu et al. [100] investigated the sleep-mode scheduling problem in order to maximize the NL by only turning on a specific subset of sensors for monitoring the target spots and for exploiting the transmission of the sensed data over multiple hops, all the way to the base station. As another design alternative, $\mathrm{Hu}$ et al. [101] employed a genetic algorithm for solving the problem of finding the maximum number of disjoint subsets of sensors for maximizing the NL,

\footnotetext{
${ }^{6} \mathrm{~A}$ specific sensor field is partitioned into smaller sensor subsets, where each subset may be composed of several sensors that are potentially closer to each other. The main idea of the disjoint subsets, also referred as the sensor covers, is to allow each sensor under the same subset to successively carry out all the tasks of that particular disjoint coverage area. More explicitly, the sensors within the same subset are not turned on at the same time. Instead, they are rather activated sequentially, after the previous sensor has run out of battery. This method assists in extending the NL.

${ }^{7}$ Barrier coverage is exploited especially in military applications, where an intruder crossing a particular region has to be detected. Therefore, a sensor barrier is usually formed by several connected sensors across the entire target region, which may feature a trip-wire-like structure to detect any potential crossings by intruders.
} 
TABLE VII

OF(S), CONSTRAINT(S) AND OPTIMIZATION ALGORITHM(S) IN THE CONTEXT OF MOBILE RELAY AND/OR SINK TECHNIQUES THAT MAXIMIZE THE LIFETIME OF WSNS.

\begin{tabular}{|c|c|c|c|c|}
\hline Year & Author(s) & OF(s) & Constraint function(s) & Optimization tool(s) and optimality \\
\hline \multirow{3}{*}{2008} & $\begin{array}{l}\text { Wang et } \\
\text { al. } \text { [54] }\end{array}$ & NL-1: NL & $\begin{array}{l}\text { Const.-2,-6: Resource rich mo- } \\
\text { bile relay sensors, assisting heav- } \\
\text { ily burdened sensors, routing }\end{array}$ & $\begin{array}{l}\text { Joint mobility of relay nodes and aggregation } \\
\text { routing algorithm [150]. Near-optimal solution is } \\
\text { obtained. }\end{array}$ \\
\hline & $\begin{array}{l}\text { Hamida } \text { et } \\
\text { al. [29] }\end{array}$ & NL-12: NL & $\begin{array}{l}\text { Const.-2,-6: Mobile sink nodes, } \\
\text { overhead balancing, data dissem- } \\
\text { ination }\end{array}$ & $\begin{array}{l}\text { The geographic hash table [151], line based data } \\
\text { dissemination [152], column-row location ser- } \\
\text { vice [153]. Suboptimal solutions are obtained. }\end{array}$ \\
\hline & $\begin{array}{l}\text { Shi et } \\
\text { al. [88] }\end{array}$ & NL-1: NL & $\begin{array}{l}\text { Const.-2,-6: Mobile sink nodes, } \\
\text { routing, flow conservation, rate } \\
\text { and energy constraints }\end{array}$ & $\begin{array}{l}\text { An approximation algorithm that intelligently di- } \\
\text { vides the search space into subareas, which are } \\
\text { represented by "fictitious cost point" [154]. A } \\
\text { near-optimal solution is obtained, where NL is } \\
\text { guaranteed to be at least }(1-\varepsilon) \text { of the optimal NL } \\
\text { provided that } \varepsilon>0 \text { and can be arbitrarily small. }\end{array}$ \\
\hline 2010 & $\begin{array}{l}\text { Luo et al. } \\
{[55]}\end{array}$ & & $\begin{array}{l}\text { Const.-2,-6: Mobile sink nodes, } \\
\text { routing flow conservation, rate } \\
\text { and energy constraints }\end{array}$ & $\begin{array}{l}\text { An efficient primal-dual algorithm [136] for a } \\
\text { single mobile sink and extended duality theory } \\
\text { based approximation algorithm for multiple sinks. } \\
\text { A near-optimal solution is obtained. }\end{array}$ \\
\hline 2013 & $\begin{array}{l}\text { Yun et al. } \\
\text { [90], [91] }\end{array}$ & NL-11: NL & $\begin{array}{l}\text { Const.-2,-6: Mobile sink node, } \\
\text { delay tolerance, optimal sink po- } \\
\text { sitioning, energy and flow con- } \\
\text { servation constraints }\end{array}$ & $\begin{array}{l}\text { A subgradient algorithm based on delay tolerant } \\
\text { mobile sink model [130], [146] using GNU linear } \\
\text { programming kit (GLPK) [155]. Locally optimal } \\
\text { solution is obtained. }\end{array}$ \\
\hline 2014 & $\begin{array}{l}\text { Wang et } \\
\text { al. [89] }\end{array}$ & NL-1: NL & $\begin{array}{l}\text { Const.-2,-6: Mobile sink, sink re- } \\
\text { location, residual battery energy, } \\
\text { adaptive transmission range }\end{array}$ & $\begin{array}{l}\text { Energy-aware sink relocation algorithm adopting } \\
\text { energy-aware routing maximum capacity path. } \\
\text { Suboptimal solution is obtained. }\end{array}$ \\
\hline 2015 & $\begin{array}{l}\text { Tashtarian } \\
\text { et al. [92] }\end{array}$ & NL-1: NL & $\begin{array}{lcr}\text { Const.-2,-6: } & \text { Mobile } & \text { sink, } \\
\text { continuous and } & \text { optimal } \\
\text { trajectory (COT) } & \end{array}$ & $\begin{array}{l}\text { The COT is computed with the aid of an ap- } \\
\text { proximation algorithm. Near-optimal solution is } \\
\text { obtained. }\end{array}$ \\
\hline
\end{tabular}

where the disjoint subsets of sensors had the particular feature that each sensor of a specific subset provides full coverage of the target area. The major contributions on the subject of coverage and connectivity improvement techniques conceived for the sake of NL maximization are summarized in Fig. 10 of this section, while their OFs, constraints, optimization algorithms and optimality are surveyed in Table VIII.

Nonetheless, the sensors that are close to the sink node are often exposed to excessive tele-traffic, since these sensors have to relay data for a large number of sensors in the rest of the coverage area and hence they tend to drain their battery much more rapidly than the rest of the sensors. One way of alleviating this problem is to conceive an efficient node deployment that avoids the tele-traffic bottleneck. An optimal deployment must provide full coverage for the target area, while maintaining a reliable connectivity and best possible $\mathrm{NL}$, for example by setting the redundant sensors to their sleep-mode within the same region. In the literature, there are various optimal deployment strategies that maximize the NL. A specific example of this can be found in [102], where Natalizio et al. analyzed the optimal placement of the sensor nodes within a particular sensing field in order to maximize the NL of the WSN considered. Liu et al. [39] focused their attention on identifying the tele-traffic bottlenecks and the energy-hole regions, thus further improving the nodedeployment strategy, while achieving a balanced ED across the network was guaranteed for the sake of maximizing the NL. Similarly, a robust traffic-flow-aware scale-free topology was developed by Wang et al. [49], where the traffic-flow and hence also the ED across the network was balanced.

An appealing node-deployment strategy was proposed by Magno et al. [103], where an ultra-low-power overlay network was super-imposed on a less energy-efficient WSN in order to extend the NL of the low-efficiency WSN designed for potentially power-hungry surveillance applications supported by the low-power overlay network relying on the most recent ad- 


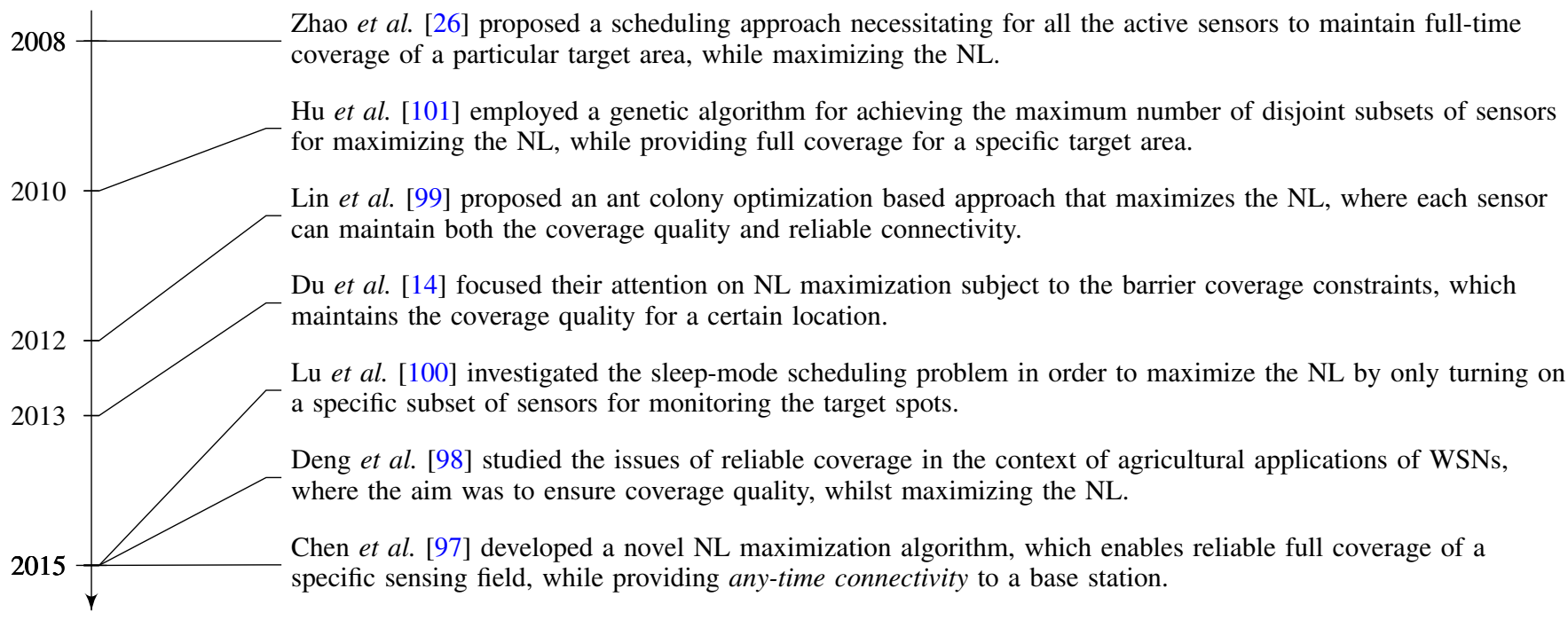

Fig. 10. Timeline of the coverage and connectivity improvement techniques designed for NL maximization.

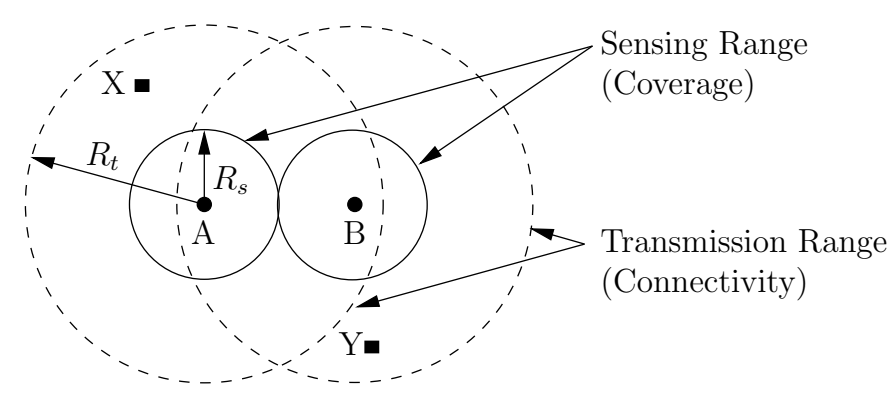

Fig. 11. The relationship between the sensing and the connectivity ranges, when $R_{t}>R_{s}$.

vances both in energy harvesting and wake-up radio technologies. Additionally, Mini et al. [104] determined the optimal deployment locations of specific sensor nodes and developed a scheduling scheme for these optimally-located sensors so that the overall NL was maximized, while achieving the required target coverage level. They also demonstrated [104] that in order to guarantee the target coverage level and to maximize the NL, only the minimum number of sensor nodes guaranteeing seamless connectivity was allowed to be scheduled, while the redundant sensors would only be used, when absolutely necessary for preventing any potential NL-expiry. More explicitly, turning on all the sensors together is energy-inefficient. Instead, turning off the sensors adjacent to the one currently operating and turning them on one-by-one, only when it is required, is capable of significantly increasing the NL, while maintaining the desired coverage probability. Similarly, Wang et al. [40] investigated the relay node placement problem under specific coverage, connectivity and NL constraints in heterogeneous WSNs. Phan et al. [38] presented a twostage cross-layer optimization technique, where the first stage involved maximizing the number of sensor nodes deployed within the existing WSN, while the second stage considered both the power allocation and scheduling operations in order to maximize the NL. As a further beneficial solution, in [105],
Cristescu et al. investigated the power efficient data gathering problem subject to particular distortion constraints, while providing the optimal node placement solution by striking a trade-off between the total power dissipation and the NL.

Najimi et al. [16] proposed a node selection algorithm for balancing the ED of the sensors in order to maximize the NL, where the sensor nodes having the highest residual battery charges are chosen for spectrum sensing in wireless cognitive sensor networks. Another deployment strategy designed for maximizing the NL was proposed in [106], [107], which relied on the cooperation of sensor nodes. On the other hand, an ant colony optimization based transmission scheme was designed for maximizing the NL by Liu et al. [108], where each sensor was capable of adjusting its transmission range for data transmission using the best possible energy efficiency and the best possible energy balancing approaches. An energy harvesting approach using a solar-powered relay node was conceived in support of the cluster head by Zhang et al. [109], where the optimal location of the cluster head was given by that maximizing the NL. The major contributions on optimal node-deployment techniques designed for NL maximization are presented in Fig. 12 of this section, while their OFs, constraints, optimization algorithms and optimality are surveyed in Table IX.

\section{F. Data Gathering and Network Coding}

One of the fundamental operations of the WSNs is to collect data from sensors and to convey it to the sink node. During the data collection stage, data aggregation can be employed to fuse data from different sensors in order to prevent redundant data transmission. More explicitly, $\mathrm{He}$ et al. [110] considered an energy-efficient cross-layer design for the gathering of spatially correlated sensory information, in order to minimize the energy-waste that would be assigned to redundant information and thus to maximize the NL. Similarly, Cristescu et al. [105] investigated power-efficient 


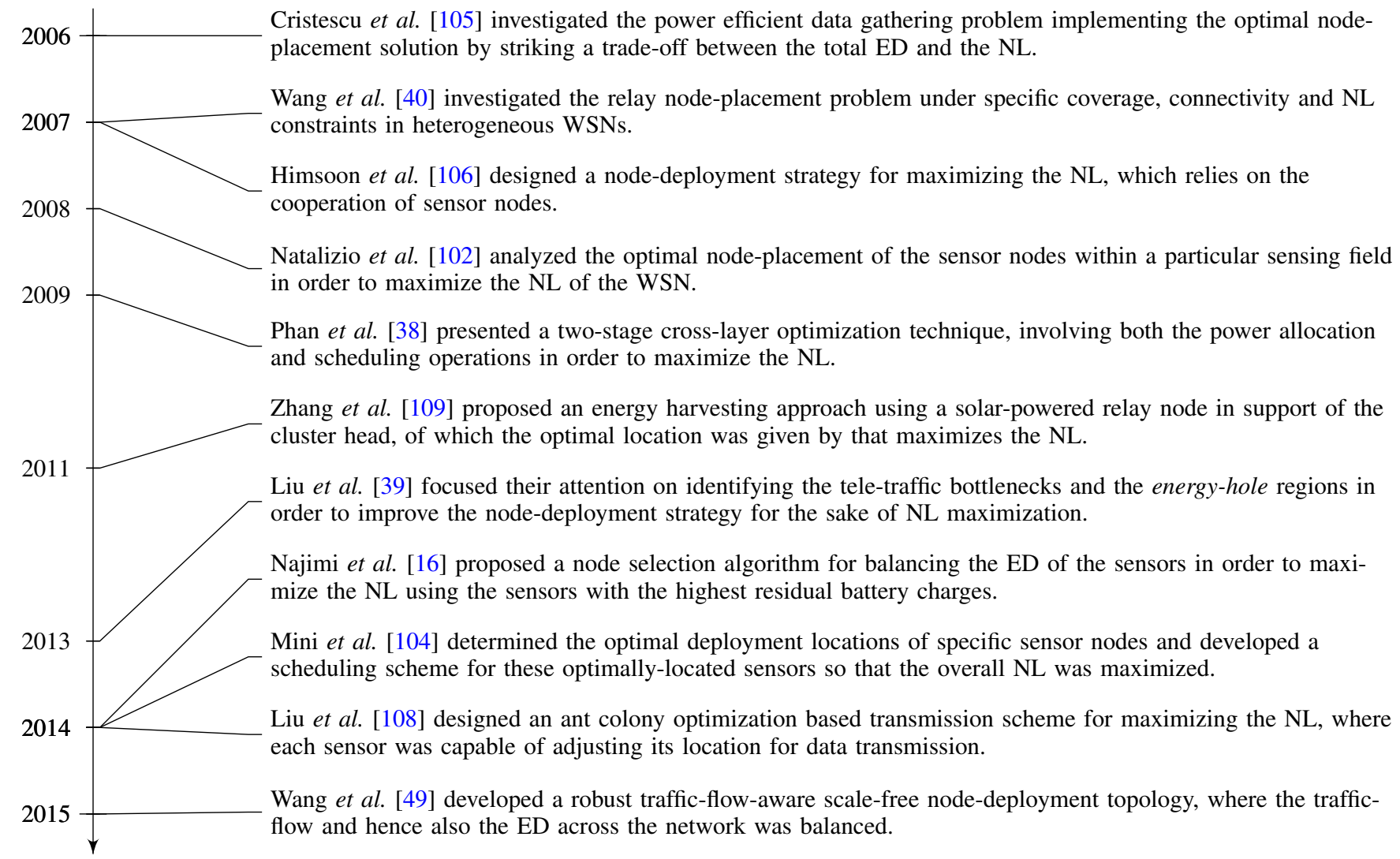

Fig. 12. Timeline of the optimal node-deployment techniques conceived for NL maximization.

data gathering subject to certain distortion constraints, while providing the optimal node placement solution subject to striking a trade-off between the total power dissipation and the NL. Additionally, Bhardwaj et al. [23], [24] focused their attention on the fundamental constraints of the information gathering and transmission to a base station, while deriving the upper bounds of the achievable NL considering the impact of several parameters on the NL, including the base station location, path loss, initial battery charge, source location and so on. In [111], Liang et al. considered an energy-efficient data gathering method constructed for maximizing the NL, where the goal was to maximize the number of data gathering queries processed, until the first node failure occurs due to exhausted battery charge in the WSN considered. Additionally, another data gathering method was proposed in [112], where a data gathering tree was constructed for the transmission of the sensed data through each sensor all the way to the base station, while preventing the formation of tele-traffic bottlenecks in order to balance the traffic-load across the network and to extend the attainable NL.

Nonetheless, network coding was designed for enabling the intermediate nodes to compress their data packets that are received from their adjacent nodes [113]. Network coding has been shown to be able to enhance the energy-efficiency of wireless networks, hence improving their NL, as discussed in [114]-[116]. Further examples include [117], where ShahMansouri et al. analyzed the trade-off between NL max- imization and minimizing the number of network coding operations, hence substantially reducing the required transmit power, albeit these signal processing operations dissipate additional power from the non-rechargeable battery. Decoding operations may also significantly reduce the NL. Similarly, Rout $e$ t al. [113] attempted to enhance the energy-efficiency of the frequently activated bottleneck nodes that are usually in the vicinity of the sink node by jointly considering sleep scheduling and network coding in order to maximize the NL.

\section{G. Data Correlation}

A salient characteristic of WSNs is that the data collected by the adjacent sensors may represent redundant information owing to the temporal-spatial data correlation characteristics of the neighboring sensors. Reducing the overall tele-traffic by removing the redundancy can be beneficial in terms of energy conservation and hence NL maximization. For example, He et al. [110] conceived an energy-efficient cross-layer design for the optimal data gathering from spatially correlated sensors in order to minimize the energy-wastage imposed by transmitting redundant information and thus to maximize the NL. Similarly, He et al. [53] proposed a method of predicting the data to be collected by a specific sensor based on the temporal-spatial correlations of its neighboring sensors, which may then lead to an extended NL, since these sensors whose data can be predicted may be turned off. Additionally, Heo et al. [118] introduced a prediction scheme for minimizing the traffic load 
TABLE VIII

OF(S), CONSTRAINT(S) AND OPTIMIZATION ALGORITHM(S) IN THE CONTEXT OF COVERAGE AND CONNECTIVITY IMPROVEMENT TECHNIQUES DESIGNED FOR MAXIMIZING THE LIFETIME OF WSNS.

\begin{tabular}{|c|c|c|c|c|}
\hline Year & Author(s) & $\mathbf{O F}(\mathbf{s})$ & Constraint function(s) & Optimization tool(s) and optimality \\
\hline 2008 & Zhao et al. [26] & NL-8: NL & $\begin{array}{l}\text { Const.-2,-3: Full-time coverage for } \\
\text { a specific area, any-time connectiv- } \\
\text { ity to sink node via a multi-hop } \\
\text { route }\end{array}$ & $\begin{array}{l}\text { A heuristic algorithm based on an approx- } \\
\text { imation algorithm called communication } \\
\text { weighted greedy cover algorithm [26] } \\
\text { Near-optimal solution is obtained. }\end{array}$ \\
\hline 2010 & Hu et al. [101] & NL-5: NL & $\begin{array}{l}\text { Const.-1,-2,-3: Maximum number } \\
\text { of disjoint connected sensor subsets } \\
\text { that maintain complete coverage, } \\
\text { sleep scheduling }\end{array}$ & $\begin{array}{l}\text { A hybrid genetic algorithm with schedule } \\
\text { transition operations. Suboptimal solution } \\
\text { is obtained. }\end{array}$ \\
\hline 2012 & Lin et al. [99] & NL-8: NL & $\begin{array}{l}\text { Const.-2,-3: Maximum number of } \\
\text { disjoint connected sensor subsets } \\
\text { that maintain sensing coverage and } \\
\text { network connectivity }\end{array}$ & $\begin{array}{l}\text { Ant colony optimization based approach } \\
\text { for maximizing the number of connected } \\
\text { sensor subsets. Suboptimal solution is ob- } \\
\text { tained. }\end{array}$ \\
\hline 2013 & Du et al. [14] & NL-1: NL & $\begin{array}{l}\text { Const.-2,-3,-6: Redeployment, } \\
\text { sleep scheduling, } k \text {-discrete barrier } \\
\text { coverage probability }\end{array}$ & $\begin{array}{l}\text { Maximum-lifetime for } k \text {-discrete barrier } \\
\text { coverage with limited-moving cost algo- } \\
\text { rithm [14]. Suboptimal solution is ob- } \\
\text { tained. }\end{array}$ \\
\hline \multirow{3}{*}{2015} & Lu et al. [100] & NL-6: NL & $\begin{array}{l}\text { Const.-1,-2,-3: Sleep scheduling, } \\
\text { target coverage, data collection, } \\
\text { multi-hop communication }\end{array}$ & $\begin{array}{l}\text { Maximum lifetime coverage scheduling } \\
\text { problem is solved using polynomial- } \\
\text { time constant-factor approximation algo- } \\
\text { rithm [156]. Near optimal solution is ob- } \\
\text { tained. }\end{array}$ \\
\hline & Deng et al. [98] & NL-8: NL & $\begin{array}{l}\text { Const.-1,-2,-3: Confident informa- } \\
\text { tion coverage, activity scheduling }\end{array}$ & $\begin{array}{l}\text { Multi-modal confident information cover- } \\
\text { age problem solved via both a centralized } \\
\text { and a distributed heuristic algorithm. Sub- } \\
\text { optimal solution is obtained. }\end{array}$ \\
\hline & Chen et al. [97] & NL-4: NL & $\begin{array}{l}\text { Const.-1,-2,-3: Least number of } \\
\text { sensor activation, traffic-balanced } \\
\text { routing, full-time coverage, any- } \\
\text { time connectivity }\end{array}$ & $\begin{array}{l}\text { Maximum connected load-balancing } \\
\text { cover tree algorithm based on heuristic } \\
\text { coverage control and traffic balanced } \\
\text { routing strategy [97]. Suboptimal solution } \\
\text { is obtained. }\end{array}$ \\
\hline
\end{tabular}

across the WSN, which was further minimized by taking advantage of the spatial correlation of the various sensors in the interest of maximizing the NL. They demonstrated in [118] that the amount of data to be transmitted can be reduced by $20 \%$ using the proposed scheme by exploiting both the context prediction and the spatial correlation amongst the sensors, which hence extended the NL.

The joint optimization of the data aggregation and maximum lifetime-based routing was considered in [78], where data aggregation reduces the traffic-load across the network by taking advantage of the temporal-spatial data correlation. As a benefit, the power dissipation of the sensor nodes that are adjacent to the sink node can be substantially reduced and then the ensuing maximum lifetime-based routing further balances the tele-traffic for the sake of avoiding any potential bottleneck formation.

\section{H. Energy Harvesting}

Energy harvesting devices have been conceived for scavenging energy from the environment, hence they are often referred to as energy harvesting wireless sensor networks (EHWSNs) [119], [120]. Compared to conventional battery powered WSNs, EH-WSNs provide substantial benefits in terms of NL maximization [121]-[123]. However, from a practical point of view, the entire WSNs cannot purely rely on nodes equipped with EH devices due to the high cost and owing to 
TABLE IX

OF(S), CONSTRAINT(S) AND OPTIMIZATION ALGORITHM(S) IN THE CONTEXT OF OPTIMAL DEPLOYMENT TECHNIQUES THAT MAXIMIZE THE LIFETIME OF WSNS.

\begin{tabular}{|c|c|c|c|c|}
\hline Year & Author(s) & OF(s) & Constraint function(s) & Optimization tool(s) and optimality \\
\hline 2006 & $\begin{array}{l}\text { Cristescu } \\
\text { et al. }[105]\end{array}$ & $\begin{array}{l}\text { NL-1: NL, } \\
\text { total power } \\
\text { dissipation }\end{array}$ & $\begin{array}{l}\text { Const.-1,-2,-6: Optimal transmission } \\
\text { scheme, optimal node placement, rate } \\
\text { allocation, data gathering }\end{array}$ & $\begin{array}{l}\text { An optimal placement algorithm and a } \\
\text { lifetime optimization algorithm. Near- } \\
\text { optimal solution is obtained. }\end{array}$ \\
\hline \multirow[t]{2}{*}{2007} & $\begin{array}{l}\text { Wang et } \\
\text { al. [40] }\end{array}$ & NL-1: NL & $\begin{array}{l}\text { Const.-2,-3,-6: Coverage quality, relay } \\
\text { node placement, network connectivity }\end{array}$ & $\begin{array}{l}\text { Local optimal approach for the placement } \\
\text { of the first and second phase relay nodes. } \\
\text { Optimal and near-optimal solutions are } \\
\text { provided. }\end{array}$ \\
\hline & $\begin{array}{l}\text { Himsoon et } \\
\text { al. [106] }\end{array}$ & NL-1: NL & $\begin{array}{l}\text { Const.-2,-5,-6: Cooperative diversity, } \\
\text { BER constraint, node selection, power } \\
\text { allocation, optimal deployment }\end{array}$ & $\begin{array}{l}\text { A reduced complexity suboptimal algo- } \\
\text { rithm. }\end{array}$ \\
\hline 2008 & $\begin{array}{l}\text { Natalizio et } \\
\text { al. [102] }\end{array}$ & NL-1: NL & $\begin{array}{l}\text { Const.-2,-6: Optimal placement, } \\
\text { power control, residual battery charge }\end{array}$ & $\begin{array}{l}\text { Monte Carlo simulations. Near-optimal } \\
\text { solution is obtained. }\end{array}$ \\
\hline 2009 & $\begin{array}{l}\text { Phan et al. } \\
\text { [38] }\end{array}$ & NL-1: NL & $\begin{array}{l}\text { Const.-2,-6: Sensor node admission } \\
\text { and deployment, power allocation, } \\
\text { link scheduling }\end{array}$ & $\begin{array}{l}\text { Cross-layer optimization framework based } \\
\text { on mixed integer linear programming us- } \\
\text { ing CPLEX library [132]. Optimal solu- } \\
\text { tion is obtained. }\end{array}$ \\
\hline 2011 & $\begin{array}{l}\text { Zhang et } \\
\text { al. [109] }\end{array}$ & NL-1: NL & $\begin{array}{l}\text { Const.-2,-6: Energy harvesting solar } \\
\text { powered relay node, optimal location } \\
\text { of cluster head, clustering }\end{array}$ & $\begin{array}{l}\text { Single cluster algorithm for finding the } \\
\text { best location of cluster head. Near-optimal } \\
\text { solution is obtained. }\end{array}$ \\
\hline 2013 & $\begin{array}{ll}\text { Liu } & \text { et } \\
\text { al. [39] }\end{array}$ & NL-6: NL & $\begin{array}{l}\text { Const.-2,-3,-5,-6: Sensor deployment, } \\
\text { adaptive transmission range, balanced } \\
\text { ED, coverage quality, network con- } \\
\text { nectivity, avoidance of energy hole } \\
\text { regions, deployment strategy }\end{array}$ & $\begin{array}{l}\text { An algorithm based on first node die time } \\
\text { and all nodes die time NL definitions for } \\
\text { finding the optimal transmission radius } \\
\text { using OMNeT++. }\end{array}$ \\
\hline \multirow{3}{*}{2014} & $\begin{array}{l}\text { Najimi et } \\
\text { al. [16] }\end{array}$ & NL-2: NL & $\begin{array}{l}\text { Const.-2,-6: Node selection for bal- } \\
\text { anced } \mathrm{ED} \text {, maximize minimum resid- } \\
\text { ual battery charge }\end{array}$ & $\begin{array}{l}\text { An iterative algorithm using convex op- } \\
\text { timization based on Karush-Kuhn-Tucker } \\
\text { optimality. Optimal solution is obtained. }\end{array}$ \\
\hline & $\begin{array}{l}\text { Mini et } \\
\text { al. [104] }\end{array}$ & NL-6: NL & $\begin{array}{l}\text { Const.-1,-2,-3,-6: Optimal deployment } \\
\text { locations, sleep scheduling, require } \\
\text { target coverage level }\end{array}$ & $\begin{array}{l}\text { A heuristic method for sleep scheduling, } \\
\text { which can achieve the theoretical upper } \\
\text { bound of NL. }\end{array}$ \\
\hline & $\begin{array}{l}\text { Liu et } \\
\text { al. [108] }\end{array}$ & NL-1: NL & $\begin{array}{l}\text { Const.-1,-2,-4,-6: Transmission range, } \\
\text { maximum possible energy efficiency, } \\
\text { maximum possible energy balancing }\end{array}$ & $\begin{array}{l}\text { An algorithm for finding optimal trans- } \\
\text { mission scheme based on ant colony opti- } \\
\text { mization. Suboptimal solution is obtained. }\end{array}$ \\
\hline 2015 & $\begin{array}{ll}\text { Wang et } \\
\text { al. [49] }\end{array}$ & NL-1: NL & $\begin{array}{l}\text { Const.-2,-3,-4,-6: Balanced ED and } \\
\text { traffic flow, connectivity, robustness } \\
\text { against node failure, energy-efficient } \\
\text { topology }\end{array}$ & $\begin{array}{l}\text { Flow-aware scale-free topology model an- } \\
\text { alyzed using shortest path and low-energy } \\
\text { adaptive clustering hierarchy [157] algo- } \\
\text { rithms. Suboptimal solution is obtained. }\end{array}$ \\
\hline
\end{tabular}


a range of other physical constraints of EH sensor devices. As an intermediate solution, a solar-powered node was used as the cluster head in [109], where the optimal location of the cluster head was determined using a specific cluster scheme conceived for lifetime optimization. De-Witt et al. [124] incorporated energy harvesting into the barrier coverage problem investigated in [14] and developed a certain solution to the problem of maximizing the lifetime of $k$-barrier coverage in EH-WSNs, while Martinez et al. [125] incorporated the energy harvesting capability and the energy storage capacity limits into the associated routing decisions. Nonetheless, Tabassum et al. [119] argues that achieving the required QoS for battery-constrained wireless applications can be challenging due to battery failures, which can be compensated by energy harvesting from ambient sources. Therefore, Tabassum et al. reveals the key challenges of designing energy harvesting cellular networks in [119] in order to guarantee an increased battery-lifetime for wireless devices. By contrast, He et al. [120] surveys various methods of harvesting the ambient energy, with an emphasis on optimal offline policies.

\section{Beamforming}

Distributed or collaborative beamforming utilizes multiple single-antenna-aided transmitters, which form distributed antenna arrays, whose phase-coherently combined waves create angularly selective beams directed at the intended receiver, which significantly increases the transmission distance. Each transmitter can reduce its transmit power, since the ED is shared amongst several transmitters. However, consistently utilizing the same transmitters may completely drain the battery charge of these specific sensors. Therefore, the failing transmitters may lead to a coverage degradation in a particular area. A beneficial beamforming solution was provided by Feng et al. [126], where the authors explored the factors influencing the ED and the NL. Feng et al. [126] also proposed an algorithm providing a carefully balanced selection of the transmitters for maximizing the NL, where the NL is doubled compared to direct or multihop transmissions through a particular receiver that is located far-away from the sensing field. Similarly, Bejar-Haro et al. [30] designed an energy-efficient collaborative beamforming scheme for transmitting data to a far-away base station for the sake of NL maximization, while satisfying the target QoS requirement, such as the SNR requirement. Additionally, Han et al. [127] aimed for maximizing the NL by exploiting collaborative beamforming and cooperative transmission techniques that can be invoked by the closely located sensors in order to reduce the trafficload and to prevent the relaying of data by the specific sensors having critical battery charges. The NL can be improved by $10 \%$ to $90 \%$ using the transmission technique of [127] depending on the particular network topology considered.

\section{SUMMARY}

Bearing in mind the contributions on the NL maximization problem considered, we summarize our findings, including some conclusive design guidelines, the lessons learned and future research directions, as follows.

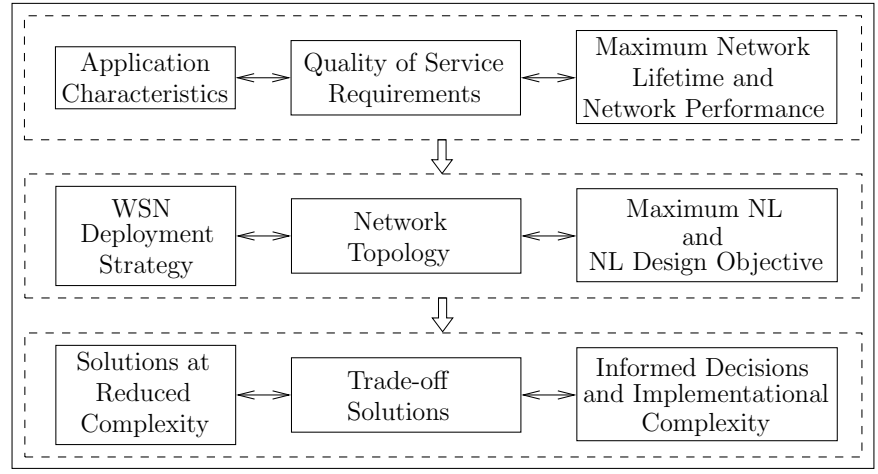

Fig. 13. Design criteria of energy-constrained WSNs in the interest of maximizing the NL.

\section{A. Conclusions}

In the following, we formulate some design guidelines for constructing maximum-lifetime applications of WSNs, as portrayed in Fig. 13.

1. QoS Requirements: Observe in Fig. 13 that determining the characteristics of the application considered and its QoS requirements as well as the network's design constraints play a vital role in terms of maximizing the performance of the WSN. For example, video surveillance applications require higher data rate for maintaining the desired QoE of the user(s). On the other hand, these applications may necessitate a higher transmit power, where the NL may be significantly degraded, since the sensors may be exposed to an increased interference. For such an application, the system designer has to define the lowest acceptable QoS, whilst attaining a longer operational time for the WSN considered. Nonetheless, different design constraints tend to require different strategies for NL maximization. For example, maximizing the NL, while maintaining the desired coverage quality and network connectivity requires different considerations than NL maximization, while maintaining an optimal sleep scheduling scheme and opportunistic routing strategy.

2. NL Design Objective: Once the application requirements have been determined, the WSN deployment strategy has to be specified, which ultimately determines the network topology that is vitally important for NL maximization. Explicitly, for example a network having a string topology, where only the adjacent nodes are within each others' transmission range, the NL is strictly dependent on that particular node's lifetime, which completely depletes its battery. However, in a network having numerous alternative routes, the NL may be dependent only on the SN's lifetime, since the sensor measurements can be delivered over numerous alternative routes. Therefore, the NL can be defined depending on the particular application and on its network topology.

3. Computational Complexity: Having described the application characteristics and its QoS requirements along with the NL definition that relies on the network topology constructed, finding optimal solutions for the sake of NL maximization, at a reduced complexity and/or providing trade-off solutions between several important objective functions is vitally important for attaining the optimal communication parameter 
values at a reduced implementational complexity. It is also of salient importance to provide the system designer with a well-informed decision for the ensuing hardware implementations by carefully balancing the interplay amongst several conflicting objectives, while guaranteeing the desired QoS requirements.

4. Design Criteria Models for the Lifetime Maximization of WSNs: In [9] Madan et al. considered the jointly optimized transmit rate, power and link scheduling for the sake of NL maximization in an interference-limited WSN communicating over an AWGN channel. They also demonstrated the benefits of multi-hop routing, traffic-load balancing, interference management and spatial reuse in extending the NL. In addition to this, we analyzed the impact of the poor channel conditions on the NL in the face of fading channels [11]. The design criteria of Fig. 13 were partially inferred from [9] and [11] for the sake of NL maximization, as illustrated in Table X.

Furthermore, Gu et al. [158] studied the options of beneficial base station placement with the objective of extending the NL based on a specific problem formulation, given specific flow routing and energy conservation constraints. A heuristic algorithm was proposed for solving the NL maximization problem at a reduced complexity, albeit this was achieved at the cost of a small reduction in NL compared the optimal NL solution. On the other hand, in [13] we proposed a two-stage NL maximization technique, where the NL was dependent on the SN's residual battery, since the SN's information was transmitted to a DN over alternative routes of a highcomplexity fully-connected WSN. Each route used for the transmission of the SN's information was computed for its route lifetime (RL) and these RL values were computed relying on the residual battery levels that were summed in order to determine the overall NL, until the SN's battery was completely depleted. The exhaustive search algorithm (ESA) was proposed for obtaining the optimal NL solution at the cost of a higher computational complexity and a single-objective genetic algorithm (SOGA) was developed for achieving a near-optimal solution at a significantly reduced complexity compared to ESA. Our design criteria of Fig. 13 are also in line with those of [158] and [13] in the interest of maximizing the NL, as seen in Table X.

\section{B. Lessons Learned}

The family of NL maximization techniques has attracted a lot of research attention for the sake of prolonging the flawless operation of battery-constrained WSNs. Therefore, in this treatise we have outlined the design constraints of WSNs in the interest of extending the NL. Commencing with the portrayal of rich variety definitions of NL design objective used for WSNs, the family of NL maximization techniques was introduced. A range of design guidelines illustrated by examples has been provided in order to characterize the potential improvements of the different design criteria. We have demonstrated that the design constraints, definitions of NL design objective and NL maximization techniques have to be carefully selected depending on the specific application and on the objective function to be optimized.
We were able to classify NL maximization techniques into resource allocation relying on cross-layer design as well as into opportunistic transmission schemes and sleep-wake scheduling, routing and clustering, mobile relays and sinks, coverage and connectivity as well as optimal node-deployment, data gathering and network coding, data correlation, energy harvesting and beamforming aspects, as highlighted in this paper. We note that some of the papers may be classified into multiple NL maximization techniques. We circumvented this ambiguity by classifying the papers according to their specific context.

It is also plausible upon designing an energy-constrained WSN, the designer first has to identify the QoS requirements. Then, depending on the specific QoS requirements and objective function, the most suitable NL definition has to be determined. Finally, finding solutions at a reduced-complexity is a challenging but an important factor for informing the system designer before embarking on hardware implementations.

\section{Future Research Ideas}

We propose several directions for future research. Energy harvesting [159]-[163] is a relatively new concept in wireless sensor networks, where a sensor has the capability to convert various forms of environmental energy into electricity in order to supply the sensor node. Therefore, power allocation strategies using energy harvesting sensors can be studied [164][168] in order to extend the NL. Assuming that each sensor has a limited battery capacity, using an external energy source from nature can help in prolonging the NL. However, considering the relatively low efficiency of energy harvesters [159], the NL maximization and power allocation mechanisms still play a significant role in keeping the network functional for an extended duration. This beneficial contribution of energy harvesters in extending the NL can be formulated as part of an optimization problem, as demonstrated in [109], [169]. As part of the solution, the key challenges of designing energy harvesting aided cellular networks discussed in [119] may be taken into consideration in order to guarantee the increased battery-lifetime of wireless devices.

The mobility models described in [54], [170] can be exploited in order to study how these models affect both the convergence of the algorithms as well as the lifetime of WSNs.

Amplifying or decoding and remodulating the signals before forwarding them is capable of achieving energy savings, as discussed in [171]-[173]. Network coding also has substantial benefits [113], [115], [174]-[177] in terms of energy savings, hence extending the lifetime of WSNs.

Although the parameters of the problem formulations are mostly assumed to be constant, in practice these parameters are based on inaccurate estimates. Therefore, robust optimization can be used for mitigating the effects of unavoidable errors imposed, for example by channel estimation and power control errors [178], [179].

\section{REFERENCES}

[1] I. Akyildiz, W. Su, Y. Sankarasubramaniam, and E. Cayirci, "A survey on sensor networks," IEEE Communications Magazine, vol. 40, no. 8, pp. 102-114, August 2002. 
TABLE $X$

DESIGN CRITERIA MODELS FOR LIFETIME MAXIMIZATION OF WSNS.

\begin{tabular}{|l|l|l|l|}
\hline $\begin{array}{c}\text { Design Criteria } \\
\text { Examples }\end{array}$ & \multicolumn{1}{|c|}{$\begin{array}{c}\text { QoS } \\
\text { Requirements }\end{array}$} & \multicolumn{1}{c|}{$\begin{array}{c}\text { NL } \\
\text { Definitions }\end{array}$} & \multicolumn{1}{c|}{$\begin{array}{c}\text { Computational } \\
\text { Complexity }\end{array}$} \\
\hline \hline Yetgin et al. [11] & $\begin{array}{l}\text { Source rate, NL, } \\
\text { transmit rate, } \\
\text { transmit power }\end{array}$ & $\begin{array}{l}\text { The earliest node } \\
\text { depletes its battery }\end{array}$ & $\begin{array}{l}\text { Significantly lower } \\
\text { computational complexity } \\
\text { compared to [9] }\end{array}$ \\
\hline Madan et al. $[9]$ & $\begin{array}{l}\text { Source rate, NL, } \\
\text { transmit rate, } \\
\text { transmit power }\end{array}$ & $\begin{array}{l}\text { The earliest node } \\
\text { depletes its battery }\end{array}$ & $\begin{array}{l}\text { High complexity } \\
\text { for fading scenario }\end{array}$ \\
\hline Yetgin et al. $[13]$ & NL, SINR, BER & $\begin{array}{l}\text { The earliest node } \\
\text { depletes its battery }\end{array}$ & $\begin{array}{l}\text { Lower complexity } \\
\text { using genetic algorithm }\end{array}$ \\
\hline Gu et al. [158] & NL & $\begin{array}{l}\text { The earliest node } \\
\text { depletes its battery }\end{array}$ & $\begin{array}{l}\text { Lower complexity } \\
\text { using heuristic algorithm }\end{array}$ \\
\hline
\end{tabular}

[2] - "Wireless sensor networks: A survey," Computer Networks, vol. 38, no. 4, pp. 393-422, March 2002.

[3] K. Romer and F. Mattern, "The design space of wireless sensor networks," IEEE Wireless Communications, vol. 11, no. 6, pp. 54-61, December 2004

[4] D. Puccinelli and M. Haenggi, "Wireless sensor networks: Applications and challenges of ubiquitous sensing," IEEE Circuits and Systems Magazine, vol. 5, no. 3, pp. 19-31, September 2005.

[5] V. Gungor and G. Hancke, "Industrial wireless sensor networks: Challenges, design principles, and technical approaches," IEEE Transactions on Industrial Electronics, vol. 56, no. 10, pp. 4258-4265, October 2009.

[6] T. Igoe, Making things talk: Practical methods for connecting physical objects. O'reilly, 2007.

[7] L. Atzori, A. Iera, and G. Morabito, "The internet of things: A survey," Computer Networks, vol. 54, no. 15, pp. 2787-2805, October 2010.

[8] Y. Chen and Q. Zhao, "On the lifetime of wireless sensor networks," IEEE Communications Letters, vol. 9, no. 11, pp. 976-978, November 2005.

[9] R. Madan, S. Cui, S. Lall, and A. Goldsmith, "Cross-layer design for lifetime maximization in interference-limited wireless sensor networks," IEEE Transactions on Wireless Communications, vol. 5, no. 11, pp. 3142-3152, November 2006.

[10] J. W. Jung and M. Weitnauer, "On using cooperative routing for lifetime optimization of multi-hop wireless sensor networks: Analysis and guidelines," IEEE Transactions on Communications, vol. 61, no. 8, pp. 3413-3423, August 2013.

[11] H. Yetgin, K. T. K. Cheung, M. El-Hajjar, and L. Hanzo, "Cross-layer network lifetime maximization in interference-limited WSNs," IEEE Transactions on Vehicular Technology, vol. 64, no. 8, pp. 3795-3803, August 2015.

[12] C. Cassandras, T. Wang, and S. Pourazarm, "Optimal routing and energy allocation for lifetime maximization of wireless sensor networks with nonideal batteries," IEEE Transactions on Control of Network Systems, vol. 1, no. 1, pp. 86-98, March 2014.

[13] H. Yetgin, K. Cheung, M. El-Hajjar, and L. Hanzo, "Network-lifetime maximization of wireless sensor networks," IEEE Access, vol. 3, pp. 2191-2226, November 2015.

[14] J. Du, K. Wang, H. Liu, and D. Guo, "Maximizing the lifetime of $k$ discrete barrier coverage using mobile sensors," IEEE Sensors Journal, vol. 13 , no. 12 , pp. 4690-4701, December 2013.

[15] J. Chen, J. Li, and T. Lai, "Trapping mobile targets in wireless sensor networks: An energy-efficient perspective," IEEE Transactions on Vehicular Technology, vol. 62, no. 7, pp. 3287-3300, September 2013.

[16] M. Najimi, A. Ebrahimzadeh, S. Andargoli, and A. Fallahi, "Lifetime maximization in cognitive sensor networks based on the node selection," IEEE Sensors Journal, vol. 14, no. 7, pp. 2376-2383, July 2014.

[17] S. Soro and W. Heinzelman, "Prolonging the lifetime of wireless sensor networks via unequal clustering," in IEEE International Parallel and Distributed Processing Symposium, Denver, CO, April 2005.
[18] D. Tian and N. D. Georganas, "A coverage-preserving node scheduling scheme for large wireless sensor networks," in Proceedings of the 1st ACM international workshop on Wireless sensor networks and applications (WSNA), Atlanta, GA, USA, September 2002, pp. 32-41.

[19] W. Mo, D. Qiao, and Z. Wang, "Mostly-sleeping wireless sensor networks: Connectivity, $k$-coverage, and $\alpha$-lifetime," in Proceedings of the 43rd Annual Allerton Conference on Communication, Control and Computing, Monticello, Illinois, USA, September 2005.

[20] M. Cardei, M. T. Thai, Y. Li, and W. Wu, "Energy-efficient target coverage in wireless sensor networks," in 24th Annual Joint Conference of the IEEE Computer and Communications Societies (INFOCOM'05), vol. 3, Miami, FL, USA, March 2005, pp. 1976-1984.

[21] H. Zhang and J. C. Hou, "On the upper bound of $\alpha$-lifetime for large sensor networks," ACM Transactions on Sensor Networks, vol. 1, no. 2, pp. 272-300, November 2005.

[22] K. Wu, Y. Gao, F. Li, and Y. Xiao, "Lightweight deployment-aware scheduling for wireless sensor networks," Mobile networks and applications, vol. 10, no. 6, pp. 837-852, December 2005.

[23] M. Bhardwaj, T. Garnett, and A. P. Chandrakasan, "Upper bounds on the lifetime of sensor networks," in IEEE International Conference on Communications (ICC'01), vol. 3, Helsinki, Finland, June 2001, pp. 785-790.

[24] M. Bhardwaj and A. P. Chandrakasan, "Bounding the lifetime of sensor networks via optimal role assignments," in IEEE International Conference on Computer Communications (INFOCOM'02), vol. 3, NY, USA, June 2002, pp. 1587-1596.

[25] B. Cărbunar, A. Grama, J. Vitek, and O. Cărbunar, "Redundancy and coverage detection in sensor networks," ACM Transactions on Sensor Networks (TOSN), vol. 2, no. 1, pp. 94-128, February 2006.

[26] Q. Zhao and M. Gurusamy, "Lifetime maximization for connected target coverage in wireless sensor networks," IEEE/ACM Transactions on Networking, vol. 16, no. 6, pp. 1378-1391, December 2008.

[27] S. Baydere, Y. Safkan, and O. Durmaz, "Lifetime analysis of reliable wireless sensor networks," IEICE transactions on communications, vol. 88, no. 6, pp. 2465-2472, June 2005.

[28] J. Li and G. Al-Regib, "Network lifetime maximization for estimation in multihop wireless sensor networks," IEEE Transactions on Signal Processing, vol. 57, no. 7, pp. 2456-2466, July 2009.

[29] E. B. Hamida and G. Chelius, "Strategies for data dissemination to mobile sinks in wireless sensor networks," IEEE Wireless Communications, vol. 15, no. 6, pp. 31-37, December 2008.

[30] B. Bejar Haro, S. Zazo, and D. Palomar, "Energy efficient collaborative beamforming in wireless sensor networks," IEEE Transactions on Signal Processing, vol. 62, no. 2, pp. 496-510, January 2014.

[31] I. Dietrich and F. Dressler, "On the lifetime of wireless sensor networks," ACM Transactions on Sensor Networks, vol. 5, no. 1, pp. 1-39, February 2009

[32] W. Liu, K. Lu, J. Wang, G. Xing, and L. Huang, "Performance analysis of wireless sensor networks with mobile sinks," IEEE Transactions on Vehicular Technology, vol. 61, no. 6, pp. 2777-2788, July 2012.

[33] H. Salarian, K. Chin, and F. Naghdy, "An energy-efficient mobile-sink path selection strategy for wireless sensor networks," IEEE Transac- 
tions on Vehicular Technology, vol. 63, no. 5, pp. 2407-2419, June 2014.

[34] J. Li and G. Al-Regib, "Function-based network lifetime for estimation in wireless sensor networks," IEEE Signal Processing Letters, vol. 15, pp. 533-536, July 2008.

[35] A. Aziz, Y. Sekercioglu, P. Fitzpatrick, and M. Ivanovich, "A survey on distributed topology control techniques for extending the lifetime of battery powered wireless sensor networks," IEEE Communications Surveys and Tutorials, vol. 15, no. 1, pp. 121-144, February 2013.

[36] H. Yetgin, K. T. K. Cheung, M. El-Hajjar, and L. Hanzo, "Crosslayer network lifetime optimisation considering transmit and signal processing power in wireless sensor networks," IET Wireless Sensor Systems, vol. 4, no. 4, pp. 176-182, December 2014.

[37] W. Xu, Q. Shi, X. Wei, Z. Ma, X. Zhu, and Y. Wang, "Distributed optimal rate-reliability-lifetime tradeoff in time-varying wireless sensor networks," IEEE Transactions on Wireless Communications, vol. 13 , no. 9, pp. 4836-4847, September 2014.

[38] K. Phan, R. Fan, H. Jiang, S. Vorobyov, and C. Tellambura, "Network lifetime maximization with node admission in wireless multimedia sensor networks," IEEE Transactions on Vehicular Technology, vol. 58, no. 7 , pp. 3640-3646, September 2009

[39] A. Liu, X. Jin, G. Cui, and Z. Chen, "Deployment guidelines for achieving maximum lifetime and avoiding energy holes in sensor network," Information Sciences, vol. 230, pp. 197-226, May 2013.

[40] Q. Wang, K. Xu, G. Takahara, and H. Hassanein, "Device placement for heterogeneous wireless sensor networks: Minimum cost with lifetime constraints," IEEE Transactions on Wireless Communications, vol. 6 , no. 7, pp. 2444-2453, July 2007.

[41] J.-W. Lin and Y.-T. Chen, "Improving the coverage of randomized scheduling in wireless sensor networks," IEEE Transactions on Wireless Communications, vol. 7, no. 12, pp. 4807-4812, December 2008.

[42] R. Madan and S. Lall, "Distributed algorithms for maximum lifetime routing in wireless sensor networks," IEEE Transactions on Wireless Communications, vol. 5, no. 8, pp. 2185-2193, August 2006.

[43] M. Cheng, X. Gong, and L. Cai, "Joint routing and link rate allocation under bandwidth and energy constraints in sensor networks," IEEE Transactions on Wireless Communications, vol. 8, no. 7, pp. 37703779, July 2009.

[44] H. Wang, N. Agoulmine, M. Ma, and Y. Jin, "Network lifetime optimization in wireless sensor networks," IEEE Journal on Selected Areas in Communications, vol. 28, no. 7, pp. 1127-1137, September 2010.

[45] H. Kwon, T. H. Kim, S. Choi, and B. G. Lee, "Lifetime maximization under reliability constraint via cross-layer strategy in wireless sensor networks," in IEEE Wireless Communications and Networking Conference (WCNC'05), vol. 3, New Orleans, LA, March 2005, pp. 18911896

[46] _ "Cross-layer lifetime maximization under reliability and stability constraints in wireless sensor networks," in IEEE International Conference on Communications (ICC'05), vol. 5, Seoul, Korea, May 2005, pp. 3285-3289.

[47] _ "A cross-layer strategy for energy-efficient reliable delivery in wireless sensor networks," IEEE Transactions on Wireless Communications, vol. 5, no. 12, pp. 3689-3699, December 2006.

[48] M. Di Francesco, G. Anastasi, M. Conti, S. Das, and V. Neri, "Reliability and energy-efficiency in IEEE 802.15.4/ZigBee sensor networks: An adaptive and cross-layer approach," IEEE Journal on Selected Areas in Communications, vol. 29, no. 8, pp. 1508-1524, September 2011.

[49] D. Wang, E. Liu, Z. Zhang, R. Wang, S. Zhao, X. Huang, and F. Liu, "A flow-weighted scale-free topology for wireless sensor networks," IEEE Communications Letters, vol. 19, no. 2, pp. 235-238, February 2015.

[50] S. Raghuwanshi and A. Mishra, "A self-adaptive clustering based algorithm for increased energy-efficiency and scalability in wireless sensor networks," in IEEE 58th Vehicular Technology Conference (VTC'03-Fall), Orlando, Florida, USA, October 2003.

[51] K. Phan, H. Jiang, C. Tellambura, S. Vorobyov, and R. Fan, "Joint medium access control, routing and energy distribution in multi-hop wireless networks," IEEE Transactions on Wireless Communications, vol. 7, no. 12, pp. 5244-5249, December 2008

[52] L. Hanzo, M. El-Hajjar, and O. Alamri, "Near-capacity wireless transceivers and cooperative communications in the MIMO era: Evolution of standards, waveform design, and future perspectives," Proceedings of the IEEE, vol. 99, no. 8, pp. 1343-1385, August 2011.

[53] S. He, J. Chen, X. Li, X. Shen, and Y. Sun, "Leveraging prediction to improve the coverage of wireless sensor networks," IEEE Transactions on Parallel and Distributed Systems, vol. 23, no. 4, pp. 701-712, April 2012.

[54] W. Wang, V. Srinivasan, and K.-C. Chua, "Extending the lifetime of wireless sensor networks through mobile relays," IEEE/ACM Transactions on Networking, vol. 16, no. 5, pp. 1108-1120, October 2008.

[55] J. Luo and J.-P. Hubaux, "Joint sink mobility and routing to maximize the lifetime of wireless sensor networks: The case of constrained mobility," IEEE/ACM Transactions on Networking, vol. 18, no. 3, pp. 871-884, June 2010

[56] L. Van Hoesel, T. Nieberg, J. Wu, and P. J. M. Havinga, "Prolonging the lifetime of wireless sensor networks by cross-layer interaction," IEEE Wireless Communications, vol. 11, no. 6, pp. 78-86, December 2004.

[57] H. Nama, M. Chiang, and N. Mandayam, "Utility-lifetime tradeoff in self-regulating wireless sensor networks: A cross-layer design approach," in IEEE International Conference on Communications (ICC'06), vol. 8, Istanbul, Turkey, June 2006, pp. 3511-3516.

[58] R. Madan, S. Cui, S. Lall, and A. Goldsmith, "Modeling and optimization of transmission schemes in energy-constrained wireless sensor networks," IEEE/ACM Transactions on Networking, vol. 15, no. 6, pp. 1359-1372, December 2007.

[59] J. Zhu, S. Chen, B. Bensaou, and K.-L. Hung, "Tradeoff between lifetime and rate allocation in wireless sensor networks: A cross layer approach," in 26th IEEE International Conference on Computer Communications (INFOCOM'07), Anchorage, Alaska, USA, May 2007, pp. 267-275.

[60] J. Li, S. Dey, and J. Evans, "Maximal lifetime power and rate allocation for wireless sensor systems with data distortion constraints," IEEE Transactions on Signal Processing, vol. 56, no. 5, pp. 2076-2090, May 2008.

[61] J. Luo, A. Iyer, and C. Rosenberg, "Throughput-lifetime trade-offs in multihop wireless networks under an SINR-based interference model," IEEE Transactions on Mobile Computing, vol. 10, no. 3, pp. 419-433, March 2011.

[62] S. Ehsan, B. Hamdaoui, and M. Guizani, "Radio and medium access contention aware routing for lifetime maximization in multichannel sensor networks," IEEE Transactions on Wireless Communications, vol. 11, no. 9, pp. 3058-3067, September 2012.

[63] J.-H. Jeon, H.-J. Byun, and J.-T. Lim, "Joint contention and sleep control for lifetime maximization in wireless sensor networks," IEEE Communications Letters, vol. 17, no. 2, pp. 269-272, February 2013.

[64] S.-J. Kim, X. Wang, and M. Madihian, "Distributed joint routing and medium access control for lifetime maximization of wireless sensor networks," IEEE Transactions on Wireless Communications, vol. 6, no. 7, pp. 2669-2677, July 2007.

[65] I. Koutsopoulos and S. Stanczak, "The impact of transmit rate control on energy-efficient estimation in wireless sensor networks," IEEE Transactions on Wireless Communications, vol. 11, no. 9, pp. 32613271, September 2012.

[66] J. Matamoros and C. Antòn-Haro, "Opportunistic power allocation and sensor selection schemes for wireless sensor networks," IEEE Transactions on Wireless Communications, vol. 9, no. 2, pp. 534-539, February 2010.

[67] Y. Chen, Q. Zhao, V. Krishnamurthy, and D. Djonin, "Transmission scheduling for optimizing sensor network lifetime: A stochastic shortest path approach," IEEE Transactions on Signal Processing, vol. 55, no. 5, pp. 2294-2309, May 2007.

[68] C. V. Phan, Y. Park, H. Choi, J. Cho, and J. G. Kim, "An energyefficient transmission strategy for wireless sensor networks," IEEE Transactions on Consumer Electronics, vol. 56, no. 2, pp. 597-605, May 2010.

[69] C.-C. Hung, K. C.-J. Lin, C.-C. Hsu, C.-F. Chou, and C.-J. Tu, "On enhancing network-lifetime using opportunistic routing in wireless sensor networks," in International Conference on Computer Communications and Networks (ICCCN'10), Zurich, August 2010, pp. 1-6.

[70] D. Wu, Y. Cai, and J. Wang, "A coalition formation framework for transmission scheme selection in wireless sensor networks," IEEE Transactions on Vehicular Technology, vol. 60, no. 6, pp. 2620-2630, July 2011.

[71] F. Liu, C.-Y. Tsui, and Y. Zhang, "Joint routing and sleep scheduling for lifetime maximization of wireless sensor networks," IEEE Transactions on Wireless Communications, vol. 9, no. 7, pp. 2258-2267, July 2010.

[72] C.-C. Hsu, M.-S. Kuo, S.-C. Wang, and C.-F. Chou, "Joint design of asynchronous sleep-wake scheduling and opportunistic routing in wireless sensor networks," IEEE Transactions on Computers, vol. 63, no. 7, pp. 1840-1846, July 2014. 
[73] Y. Zhao, J. Wu, F. Li, and S. Lu, "On maximizing the lifetime of wireless sensor networks using virtual backbone scheduling," IEEE Transactions on Parallel and Distributed Systems, vol. 23, no. 8, pp. 1528-1535, August 2012.

[74] A. Chamam and S. Pierre, "On the planning of wireless sensor networks: Energy-efficient clustering under the joint routing and coverage constraint," IEEE Transactions on Mobile Computing, vol. 8, no. 8, pp. 1077-1086, August 2009.

[75] Z. Li, Y. Peng, D. Qiao, and W. Zhang, "Joint aggregation and MAC design to prolong sensor network lifetime," in 21 st IEEE International Conference on Network Protocols (ICNP'13), Goettingen, Germany, October 2013, pp. 1-10.

[76] M. Sichitiu, "Cross-layer scheduling for power efficiency in wireless sensor networks," in IEEE International Conference on Computer Communications (INFOCOM'04), vol. 3, Hong Kong, March 2004, pp. $1740-1750$.

[77] J.-H. Chang and L. Tassiulas, "Energy conserving routing in wireless ad-hoc networks," in IEEE International Conference on Computer Communications (INFOCOM'O0), vol. 1, Tel Aviv, March 2000, pp. 22-31.

[78] C. Hua and T. Yum, "Optimal routing and data aggregation for maximizing lifetime of wireless sensor networks," IEEE/ACM Transactions on Networking, vol. 16, no. 4, pp. 892-903, August 2008.

[79] S. Amiri, P. Nasiopoulos, and V. Leung, "Collaborative routing and camera selection for visual wireless sensor networks," IET Communications, vol. 5, no. 17, pp. 2443-2450, November 2011.

[80] I. Al-Shawi, L. Yan, W. Pan, and B. Luo, "Lifetime enhancement in wireless sensor networks using fuzzy approach and A-star algorithm," IEEE Sensors Journal, vol. 12, no. 10, pp. 3010-3018, October 2012.

[81] Y. Peng, Z. Li, D. Qiao, and W. Zhang, "I2C: A holistic approach to prolong the sensor network lifetime," in IEEE International Conference on Computer Communicatons (INFOCOM'13), Turin, Italy, April 2013, pp. 2670-2678.

[82] J.-H. Chang and L. Tassiulas, "Maximum lifetime routing in wireless sensor networks," IEEE/ACM Transactions on Networking, vol. 12, no. 4, pp. 609-619, August 2004.

[83] Y. He, I. Lee, and L. Guan, "Distributed algorithms for network lifetime maximization in wireless visual sensor networks," IEEE Transactions on Circuits and Systems for Video Technology, vol. 19, no. 5, pp. 704 718, May 2009.

[84] O. Younis, M. Krunz, and S. Ramasubramanian, "Node clustering in wireless sensor networks: Recent developments and deployment challenges," IEEE Network, vol. 20, no. 3, pp. 20-25, May 2006.

[85] G. Gupta and M. Younis, "Fault-tolerant clustering of wireless sensor networks," in IEEE Wireless Communications and Networking (WCNC'03), vol. 3, New Orleans, LA, USA, March 2003, pp. 1579 1584.

[86] — "Load-balanced clustering of wireless sensor networks," in IEEE International Conference on Communications (ICC'03), vol. 3, Anchorage, AK, May 2003, pp. 1848-1852.

[87] J. Rao and S. Biswas, "Data harvesting in sensor networks using mobile sinks," IEEE Wireless Communications, vol. 15, no. 6, pp. 63-70, December 2008.

[88] Y. Shi and Y. T. Hou, "Theoretical results on base station movement problem for sensor network," in 27th IEEE International Conference on Computer Communications (INFOCOM'08), Phoenix, AZ, April 2008.

[89] C.-F. Wang, J.-D. Shih, B.-H. Pan, and T.-Y. Wu, "A network lifetime enhancement method for sink relocation and its analysis in wireless sensor networks," IEEE Sensors Journal, vol. 14, no. 6, pp. 19321943, June 2014

[90] Y.-S. Yun, Y. Xia, B. Behdani, and J. Smith, "Distributed algorithm for lifetime maximization in a delay-tolerant wireless sensor network with a mobile sink," IEEE Transactions on Mobile Computing, vol. 12, no. 10, pp. 1920-1930, October 2013

[91] Y.-S. Yun and Y. Xia, "Maximizing the lifetime of wireless sensor networks with mobile sink in delay-tolerant applications," IEEE Transactions on Mobile Computing, vol. 9, no. 9, pp. 1308-1318, September 2010

[92] F. Tashtarian, M. Hossein Yaghmaee Moghaddam, K. Sohraby, and S. Effati, "On maximizing the lifetime of wireless sensor networks in event-driven applications with mobile sinks," IEEE Transactions on Vehicular Technology, vol. 64, no. 7, pp. 3177-3189, July 2015.

[93] H. Zhang and J. C. Hou, "Maintaining sensing coverage and connectivity in large sensor networks," Ad Hoc and Sensor Wireless Networks, vol. 1, no. 1-2, pp. 89-124, March 2005.
[94] M. Liu, J. Cao, W. Lou, L.-j. Chen, and X. Li, "Coverage analysis for wireless sensor networks," in Mobile Ad-hoc and Sensor Networks, ser. Lecture Notes in Computer Science, X. Jia, J. Wu, and Y. He, Eds. Springer Berlin Heidelberg, 2005, vol. 3794, pp. 711-720.

[95] D. Li and H. Liu, "Sensor coverage in wireless sensor networks," International Journal of Sensor Networks, vol. 2, 2009.

[96] M. Cardei and J. Wu, "Energy-efficient coverage problems in wireless ad-hoc sensor networks," Computer Communications, vol. 29, no. 4 pp. 413-420, February 2006.

[97] C.-P. Chen, S. Mukhopadhyay, C.-L. Chuang, M.-Y. Liu, and J.-A. Jiang, "Efficient coverage and connectivity preservation with load balance for wireless sensor networks," IEEE Sensors Journal, vol. 15, no. 1, pp. 48-62, January 2015.

[98] X. Deng, B. Wang, W. Liu, and L. Yang, "Sensor scheduling for multi-modal confident information coverage in sensor networks," IEEE Transactions on Parallel and Distributed Systems, vol. 26, no. 3, pp. 902-913, March 2015.

[99] Y. Lin, J. Zhang, H.-H. Chung, W. H. Ip, Y. Li, and Y.-H. Shi, "An ant colony optimization approach for maximizing the lifetime of heterogeneous wireless sensor networks," IEEE Transactions on Systems, Man, and Cybernetics, Part C: Applications and Reviews, vol. 42, no. 3, pp. 408-420, May 2012.

[100] Z. Lu, W. W. Li, and M. Pan, "Maximum lifetime scheduling for target coverage and data collection in wireless sensor networks," IEEE Transactions on Vehicular Technology, vol. 64, no. 2, pp. 714-727, February 2015

[101] X.-M. Hu, J. Zhang, Y. Yu, H.-H. Chung, Y.-L. Li, Y.-H. Shi, and X.-N. Luo, "Hybrid genetic algorithm using a forward encoding scheme for lifetime maximization of wireless sensor networks," IEEE Transactions on Evolutionary Computation, vol. 14, no. 5, pp. 766-781, October 2010.

[102] E. Natalizio, V. Loscri, and E. Viterbo, "Optimal placement of wireless nodes for maximizing path lifetime," IEEE Communications Letters, vol. 12, no. 5, pp. 362-364, May 2008.

[103] M. Magno, D. Boyle, D. Brunelli, E. Popovici, and L. Benini, "Ensuring survivability of resource-intensive sensor networks through ultralow power overlays," IEEE Transactions on Industrial Informatics, vol. 10, no. 2, pp. 946-956, May 2014.

[104] S. Mini, S. Udgata, and S. Sabat, "Sensor deployment and scheduling for target coverage problem in wireless sensor networks," IEEE Sensors Journal, vol. 14, no. 3, pp. 636-644, March 2014.

[105] R. Cristescu and B. Beferull-Lozano, "Lossy network correlated data gathering with high-resolution coding," IEEE Transactions on Information Theory, vol. 52, no. 6, pp. 2817-2824, June 2006.

[106] T. Himsoon, W. Siriwongpairat, Z. Han, and K. Liu, "Lifetime maximization via cooperative nodes and relay deployment in wireless networks," IEEE Journal on Selected Areas in Communications, vol. 25, no. 2, pp. 306-317, February 2007.

[107] — - "Lifetime maximization by cooperative sensor and relay deployment in wireless sensor networks," in IEEE Wireless Communications and Networking Conference (WCNC'06), Las Vegas, NV, April 2006, pp. 439-444.

[108] X. Liu, "A transmission scheme for wireless sensor networks using ant colony optimization with unconventional characteristics," IEEE Communications Letters, vol. 18, no. 7, pp. 1214-1217, July 2014.

[109] P. Zhang, G. Xiao, and H. Tan, "A preliminary study on lifetime maximization in clustered wireless sensor networks with energy harvesting nodes," in 8th International Conference on Information, Communications and Signal Processing (ICICS'11), Singapore, December 2011.

[110] S. He, J. Chen, D. Yau, and Y. Sun, "Cross-layer optimization of correlated data gathering in wireless sensor networks," IEEE Transactions on Mobile Computing, vol. 11, no. 11, pp. 1678-1691, November 2012.

[111] W. Liang and Y. Liu, "Online data gathering for maximizing network lifetime in sensor networks," IEEE Transactions on Mobile Computing, vol. 6 , no. 1 , pp. 2-11, January 2007.

[112] Y. Wu, Z. Mao, S. Fahmy, and N. B. Shroff, "Constructing maximumlifetime data gathering forests in sensor networks," IEEE/ACM Transactions on Networking, vol. 18, no. 5, pp. 1571-1584, October 2010.

[113] R. Rout and S. Ghosh, "Enhancement of lifetime using duty cycle and network coding in wireless sensor networks," IEEE Transactions on Wireless Communications, vol. 12, no. 2, pp. 656-667, February 2013.

[114] Y. Wu, P. Chou, and S.-Y. Kung, "Minimum-energy multicast in mobile ad hoc networks using network coding," IEEE Transactions on Communications, vol. 53, no. 11, pp. 1906-1918, November 2005.

[115] L. Ding, P. Wu, Z. Pan, and X. You, "Lifetime optimization for wireless multihop networks with network coding," in International Conference 
on Wireless Communications Signal Processing (WCSP'12), Huangshan, October 2012, pp. 1-6.

[116] L. Ding, P. Wu, H. Wang, Z. Pan, and X. You, "Lifetime maximization routing with network coding in wireless multihop networks," Science China Information Sciences, vol. 56, no. 2, pp. 1-15, February 2013.

[117] V. Shah-Mansouri and V. Wong, "Lifetime-resource tradeoff for multicast traffic in wireless sensor networks," IEEE Transactions on Wireless Communications, vol. 9, no. 6, pp. 1924-1934, June 2010.

[118] T. Heo, H. Kim, J.-G. Ko, Y. Doh, J.-J. Park, J. Jun, and H. Choi, "Adaptive dual prediction scheme based on sensing context similarity for wireless sensor networks," IET Electronics Letters, vol. 50, no. 6 , pp. 467-469, March 2014.

[119] H. Tabassum, E. Hossain, A. Ogundipe, and D. I. Kim, "Wirelesspowered cellular networks: Key challenges and solution techniques," IEEE Communications Magazine, vol. 53, no. 6, pp. 63-71, June 2015.

[120] Y. He, X. Cheng, W. Peng, and G. L. Stuber, "A survey of energy harvesting communications: Models and offline optimal policies," IEEE Communications Magazine, vol. 53, no. 6, pp. 79-85, June 2015.

[121] F. Vázquez-Gallego, J. Alonso-Zarate, and L. Alonso, "Reservation dynamic frame slotted-ALOHA for wireless M2M networks with energy harvesting," in IEEE International Conference on Communications (ICC'15), London, June 2015, pp. 5985-5991.

[122] W. Liu, X. Zhou, S. Durrani, H. Mehrpouyan, and S. D. Blostein, "Energy harvesting wireless sensor networks: Delay analysis considering energy costs of sensing and transmission," IEEE Transactions on Wireless Communications, vol. 15, no. 7, pp. 4635-4650, July 2016.

[123] J. Zheng, H. Zhang, Y. Cai, R. Li, and A. Anpalagan, "Game-theoretic multi-channel multi-access in energy harvesting wireless sensor networks," IEEE Sensors Journal, vol. 16, no. 11, pp. 4587-4594, June 2016.

[124] J. De-Witt and H. Shi, "Maximizing lifetime for k-barrier coverage in energy harvesting wireless sensor networks," in IEEE Global Coтmunications Conference (GLOBECOM'14), Austin, TX, December 2014, pp. 300-304.

[125] G. Martinez, S. Li, and C. Zhou, "Multi-commodity online maximum lifetime utility routing for energy-harvesting wireless sensor networks," in IEEE Global Communications Conference (GLOBECOM'14), Austin, TX, December 2014, pp. 106-111.

[126] J. Feng, Y.-H. Lu, B. Jung, D. Peroulis, and Y. C. Hu, "Energy-efficient data dissemination using beamforming in wireless sensor networks," ACM Transactions on Sensor Networks, vol. 9, no. 3, pp. 1-31, May 2013.

[127] Z. Han and H. Poor, "Lifetime improvement of wireless sensor networks by collaborative beamforming and cooperative transmission," in IEEE International Conference on Communications (ICC'07), Glasgow, June 2007, pp. 3954-3958.

[128] H. Wang, Y. Yang, M. Ma, J. He, and X. Wang, "Network lifetime maximization with cross-layer design in wireless sensor networks," IEEE Transactions on Wireless Communications, vol. 7, no. 10, pp. 3759-3768, October 2008.

[129] Y. Zhang, J. Wu, and P. J. M. Havinga, "Implementation of an on-demand routing protocol for wireless sensor networks," in 13th International Conference on Telecommunications, Portugal, May 2006.

[130] D. Palomar and M. Chiang, "A tutorial on decomposition methods for network utility maximization," IEEE Journal on Selected Areas in Communications, vol. 24, no. 8, pp. 1439-1451, August 2006.

[131] M. Chiang, C. W. Tan, D. Palomar, D. O'Neill, and D. Julian, "Power control by geometric programming," IEEE Transactions on Wireless Communications, vol. 6, no. 7, pp. 2640-2651, July 2007.

[132] IBM's ILOG CPLEX Optimization Studio. http://www-01.ibm.com/ support/knowledgecenter/SSSA5P/welcome.

[133] J.-W. Lee, R. Mazumdar, and N. B. Shroff, "Opportunistic power scheduling for dynamic multi-server wireless systems," IEEE Transactions on Wireless Communications, vol. 5, no. 6, pp. 1506-1515, June 2006.

[134] A. Ribeiro, "Ergodic stochastic optimization algorithms for wireless communication and networking," IEEE Transactions on Signal Processing, vol. 58, no. 12, pp. 6369-6386, December 2010.

[135] M. Albulet, RF power amplifiers. Raleigh, NC: SciTech, 2001.

[136] S. P. Boyd and L. Vandenberghe, Convex optimization. Cambridge university press, 2004.

[137] J. Kim, X. Lin, N. B. Shroff, and P. Sinha, "Minimizing delay and maximizing lifetime for wireless sensor networks with anycast," IEEE/ACM Transactions on Networking, vol. 18, no. 2, pp. 515-528, April 2010.

[138] P. Wang, H. Jiang, W. Zhuang, and H. Poor, "Redefinition of maxmin fairness in multi-hop wireless networks," IEEE Transactions on
Wireless Communications, vol. 7, no. 12, pp. 4786-4791, December 2008.

[139] G. H. Polychronopoulos and J. N. Tsitsiklis, "Stochastic shortest path problems with recourse," Networks, vol. 27, no. 2, pp. 133-143, March 1996.

[140] M. C.-C. Hung, K. C.-J. Lin, C.-F. Chou, and C.-C. Hsu, "EFFORT: Energy-efficient opportunistic routing technology in wireless sensor networks," Wireless Communications and Mobile Computing, vol. 13, no. 8, pp. 760-773, June 2013.

[141] W. Saad, Z. Han, M. Debbah, A. Hjorungnes, and T. Basar, "Coalitional game theory for communication networks," IEEE Signal Processing Magazine, vol. 26, no. 5, pp. 77-97, September 2009.

[142] E. Rolland, H. Pirkul, and F. Glover, "Tabu search for graph partitioning," Annals of Operations Research, vol. 63, no. 2, pp. 209-232, April 1996.

[143] C. W. Tan, D. Palomar, and M. Chiang, "Solving nonconvex power control problems in wireless networks: Low SIR regime and distributed algorithms," in IEEE Global Telecommunications Conference (GLOBECOM'05), St. Louis, MO, November 2005

[144] W. Wallis, A Beginner's Guide to Graph Theory. Birkhäuser Boston, 2010.

[145] J.-H. Chang and L. Tassiulas, "Routing for maximum system lifetime in wireless ad-hoc networks," in Proceedings of the Annual Allerton Conference on Communication, Control and Computing, vol. 37, IL, USA, September 1999, pp. 1191-1200.

[146] A. Ribeiro, N. Sidiropoulos, and G. Giannakis, "Optimal distributed stochastic routing algorithms for wireless multihop networks," IEEE Transactions on Wireless Communications, vol. 7, no. 11, pp. 42614272, November 2008.

[147] K.-Y. Cai and L. Zhang, "Fuzzy reasoning as a control problem," IEEE Transactions on Fuzzy Systems, vol. 16, no. 3, pp. 600-614, June 2008.

[148] J. Yao, C. Lin, X. Xie, A. Wang, and C.-C. Hung, "Path planning for virtual human motion using improved A* star algorithm," in Seventh International Conference on Information Technology: New Generations (ITNG'10), Las Vegas, NV, April 2010, pp. 1154-1158.

[149] V. Rao, G. Singhal, A. Kumar, and N. Navet, "Battery model for embedded systems," in 18th International Conference on VLSI Design, Kolkata, India, January 2005, pp. 105-110.

[150] J. Luo and J.-P. Hubaux, "Joint mobility and routing for lifetime elongation in wireless sensor networks," in 24th Annual Joint Conference of the IEEE Computer and Communications Societies (INFOCOM'05), vol. 3, Miami, FL, USA, March 2005, pp. 1735-1746.

[151] S. Ratnasamy, B. Karp, L. Yin, F. Yu, D. Estrin, R. Govindan, and S. Shenker, "GHT: A geographic hash table for data-centric storage," in Proceedings of the 1st ACM international workshop on Wireless sensor networks and applications, Atlanta, Georgia, USA, September 2002, pp. 78-87.

[152] E. B. Hamida and G. Chelius, "A line-based data dissemination protocol for wireless sensor networks with mobile sink," in IEEE International Conference on Communications (ICC'08), Beijing, May 2008, pp. 2201-2205.

[153] S. M. Das, H. Pucha, and Y. C. Hu, "Performance comparison of scalable location services for geographic ad hoc routing," in 24th Annual Joint Conference of the IEEE Computer and Communications Societies (INFOCOM'05), vol. 2, Miami, FL, USA, March 2005, pp. 1228-1239.

[154] Y. Hou, Y. Shi, and H. Sherali, Applied Optimization Methods for Wireless Networks. Cambridge University Press, 2014.

[155] A. Makhorin, "GNU linear programming kit," Moscow Aviation Institute, Moscow, Russia, vol. 38, 2001.

[156] L. Ding, W. Wu, J. Willson, L. Wu, Z. Lu, and W. Lee, "Constantapproximation for target coverage problem in wireless sensor networks," in IEEE Conference on Computer Communications (INFOCOM'15), Orlando, FL, March 2012, pp. 1584-1592.

[157] W. Heinzelman, A. Chandrakasan, and H. Balakrishnan, "Energyefficient communication protocol for wireless microsensor networks," in Proceedings of the 33rd Annual Hawaii International Conference on System Sciences, Maui, HI, USA, January 2000.

[158] Y. Gu, M. Pan, and W. W. Li, "Prolonging the lifetime of large scale wireless sensor networks via base station placement," in IEEE Vehicular Technology Conference (VTC'13), Las Vegas, USA, September 2013.

[159] D. Niyato, E. Hossain, M. Rashid, and V. Bhargava, "Wireless sensor networks with energy harvesting technologies: A game-theoretic approach to optimal energy management," IEEE Wireless Communications, vol. 14, no. 4, pp. 90-96, August 2007. 
[160] P. Mitcheson, E. Yeatman, G. Rao, A. Holmes, and T. Green, "Energy harvesting from human and machine motion for wireless electronic devices," Proceedings of the IEEE, vol. 96, no. 9, pp. 1457-1486, September 2008

[161] A. Harb, "Energy harvesting: State-of-the-art," Renewable Energy, vol. 36, no. 10, pp. 2641-2654, October 2011.

[162] S. Sudevalayam and P. Kulkarni, "Energy harvesting sensor nodes: Survey and implications," IEEE Communications Surveys and Tutorials, vol. 13, no. 3, pp. 443-461, September 2011.

[163] H. Visser and R. Vullers, "RF energy harvesting and transport for wireless sensor network applications: Principles and requirements," Proceedings of the IEEE, vol. 101, no. 6, pp. 1410-1423, June 2013.

[164] A. Kansal, J. Hsu, M. B. Srivastava, and V. Raqhunathan, "Harvesting aware power management for sensor networks," in 43rd ACM/IEEE Design Automation Conference, San Francisco, CA, July 2006, pp. 651-656.

[165] O. Ozel, K. Tutuncuoglu, J. Yang, S. Ulukus, and A. Yener, "Transmission with energy harvesting nodes in fading wireless channels: Optimal policies," IEEE Journal on Selected Areas in Communications, vol. 29, no. 8, pp. 1732-1743, September 2011.

[166] K. Tutuncuoglu and A. Yener, "Optimum transmission policies for battery limited energy harvesting nodes," IEEE Transactions on Wireless Communications, vol. 11, no. 3, pp. 1180-1189, March 2012.

[167] S. Mao, M. H. Cheung, and V. Wong, "Joint energy allocation for sensing and transmission in rechargeable wireless sensor networks," IEEE Transactions on Vehicular Technology, vol. 63, no. 6, pp. 28622875, July 2014.

[168] Z. Ding, S. Perlaza, I. Esnaola, and H. Poor, "Power allocation strategies in energy harvesting wireless cooperative networks," IEEE Transactions on Wireless Communications, vol. 13, no. 2, pp. 846-860, February 2014

[169] S. Yu and C. Lee, "Lifetime maximization in mobile sensor networks with energy harvesting," in IEEE International Conference on Robotics and Automation (ICRA'11), Shanghai, China, May 2011, pp. 59115916.

[170] J. Harri, F. Filali, and C. Bonnet, "Mobility models for vehicular ad hoc networks: A survey and taxonomy," IEEE Communications Surveys and Tutorials, vol. 11, no. 4, pp. 19-41, December 2009.

[171] C. Fragouli, J. Widmer, and J.-Y. Le Boudec, "A network coding approach to energy efficient broadcasting: From theory to practice," in 25th IEEE International Conference on Computer Communications (INFOCOM'06), Barcelona, Spain, April 2006, pp. 1-11.

[172] J. Goseling, R. Matsumoto, T. Uyematsu, and J. Weber, "On the energy benefit of network coding for wireless multiple unicast," in IEEE International Symposium on Information Theory (ISIT'09), Seoul, Korea, June 2009, pp. 2567-2571.

[173] A. Keshavarz Haddad and R. Riedi, "Bounds on the benefit of network coding for wireless multicast and unicast," IEEE Transactions on Mobile Computing, vol. 13, no. 1, pp. 102-115, January 2012.

[174] H. Hosseinmardi and F. Lahouti, "Multicast lifetime maximization using network coding: A cross-layer approach," in 24th Biennial Symposium on Communications, Kingston, ON, June 2008.

[175] D. Platz, D. Woldegebreal, and H. Karl, "Random network coding in wireless sensor networks: Energy efficiency via cross-layer approach," in IEEE 10th International Symposium on Spread Spectrum Techniques and Applications (ISSSTA'08), Bologna, Italy, August 2008, pp. 654 660.

[176] W. Li, J. Li, and P. Fan, "Network coding for two-way relaying networks over Rayleigh fading channels," IEEE Transactions on Vehicular Technology, vol. 59, no. 9, pp. 4476-4488, November 2010.

[177] J. Zou, H. Xiong, C. Li, R. Zhang, and Z. He, "Lifetime and distortion optimization with joint source/channel rate adaptation and network coding-based error control in wireless video sensor networks," IEEE Transactions on Vehicular Technology, vol. 60, no. 3, pp. 1182-1194, March 2011.

[178] K. Yang, J. Huang, Y. Wu, X. Wang, and M. Chiang, "Distributed robust optimization (DRO), part I: Framework and example," Optimization and Engineering, pp. 1-33, 2008.

[179] Y. Wu, K. Yang, J. Huang, X. Wang, and M. Chiang, "Distributed robust optimization (DRO) part II: Wireless power control," Optimization and Engineering, 2010.

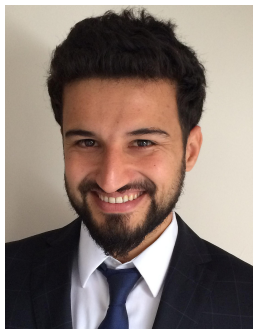

Halil Yetgin is an Assistant Professor at the department of Electrical and Electronics Engineering in Bitlis Eren University, Turkey. He received his B.Eng. degree in Computer Engineering from Selcuk University, Turkey in 2008. Then, he worked as a Software Engineer at Gulhane Military Medical Academy, Ankara, Turkey in 2009. He received his M.Sc. degree in Wireless Communications from the University of Southampton, U.K. in 2010. In 2015, he completed his Ph.D. degree in Wireless Communications at Southampton Wireless (SW) Research Group of the University of Southampton. He was a recipient of the full scholarship granted by the Republic of Turkey, Ministry of National Education. His current research interests include energy-efficient cross-layer design, multiobjective routing optimization, resource allocation of future wireless networks, bio-inspired communications, HetNETs, D2D, SON, Cellular Networks and WSNs.

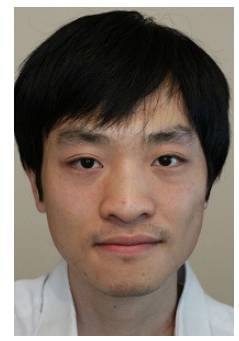

Kent Tsz Kan Cheung received his B.Eng. degree (first-class honors) in electronic engineering from the Univeristy of Southampton, Southampton, U.K., in 2009. In 2015, he completed his Ph.D. degree in wireless communications at the same institution. He was a recipient of the EPSRC Industrial CASE award in 2009, and was involved with the Core 5 Green Radio project of the Virtual Centre of Excellence in Mobile and Personal Communications (Mobile VCE). He is currently working as an engineer at Allinea Software Ltd.

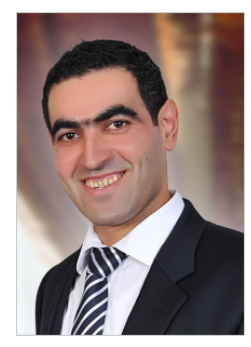

Dr. Mohammed El-Hajjar is a lecturer in the department of Electronics and Computer Science in the University of Southampton. He received his $\mathrm{PhD}$ in Wireless Communications from the University of Southampton, UK in 2008. Following the PhD, he joined Imagination Technologies as a design engineer, where he worked on designing and developing Imagination's multi-standard communications platform, which resulted in three patents. In January 2012, he joined the Department of Electronics and Computer Science in the University of Southampton as a lecturer. He is the recipient of several academic awards and has published a Wiley-IEEE book and in excess of 70 IEEE and IET journal and conference papers. Mohammed's research interests include the development of intelligent communications systems, energy-efficient transceiver design, cross-layer optimisation for large-scale networks, MIMO, millimetre wave communications and Radio over fibre systems. 


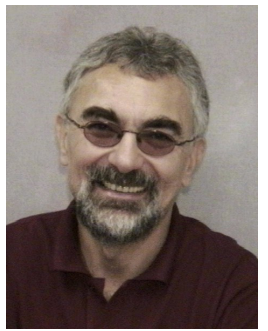

Lajos Hanzo (http://www-mobile.ecs.soton.ac.uk) FREng, FIEEE, FIET, Fellow of EURASIP, DSc received his degree in electronics in 1976 and his doctorate in 1983. In 2009 he was awarded an honorary doctorate by the Technical University of Budapest and in 2015 by the University of Edinburgh. In 2016 he was admitted to the Hungarian Academy of Science. During his 40 -year career in telecommunications he has held various research and academic posts in Hungary, Germany and the UK. Since 1986 he has been with the School of Electronics and Computer Science, University of Southampton, UK, where he holds the chair in telecommunications. He has successfully supervised 100+ $\mathrm{PhD}$ students, co-authored 18 John Wiley/IEEE Press books on mobile radio communications totalling in excess of 10000 pages, published $1600+$ research contributions at IEEE Xplore, acted both as TPC and General Chair of IEEE conferences, presented keynote lectures and has been awarded a number of distinctions. Currently he is directing a 60 -strong academic research team, working on a range of research projects in the field of wireless multimedia communications sponsored by industry, the Engineering and Physical Sciences Research Council (EPSRC) UK, the European Research Council's Advanced Fellow Grant and the Royal Society's Wolfson Research Merit Award. He is an enthusiastic supporter of industrial and academic liaison and he offers a range of industrial courses. He is also a Governor of the IEEE VTS. During 2008 - 2012 he was the Editor-in-Chief of the IEEE Press and a Chaired Professor also at Tsinghua University, Beijing. For further information on research in progress and associated publications please refer to http://wwwmobile.ecs.soton.ac.uk Lajos has $27000+$ citations and an H-index of 63. 